\title{
FINITELY VALUED COMMUTATOR SEQUENCES
}

Dedicated to the memory of Hanna Neumann

\author{
REINHOLD BAER
}

(Received 13 June 1972)

Communicated by M. F. Newman

If $x$ and $y$ are elements in the group $G$, then we denote their commutator by $x \circ y=x^{-1} y^{-1} x y=x^{-1} x^{y}$; and $x \circ G$ is the set of all commutators $x \circ g$ with $g \in G$. A $G$-commutator sequence is a series of elements $c_{i} \in G$ with $c_{1+1} \in c_{i} \circ G$. Slightly generalizing well known results one proves that the hypercenter $\mathfrak{H}_{3} G$ of the group $G$ is exactly the set of all elements $h \in G$ with the property:

every $G$-commutator sequence, containing $h$, contains 1 [Proposition 1.1].

It is clear that such a $G$-commutator sequence contains but a finite number of distinct elements. Hence we term an element $g \in G$ a $Q-G$-element, if every $G$ commutator sequence, containing $g$, is finitely valued [contains but a finite number of distinct elements]. The question arises whether the set of $Q-G$-elements is a subgroup and if so how to describe this subgroup. With this in mind denote by $\mathfrak{B G}$ the product of all the finite normal subgroups of $G$ and by $Q G$ the uniquely determined subgroup of $G$ with $\mathfrak{P} G \subseteq \mathfrak{Q} G$ and $\mathfrak{Q} G / \mathbb{B} G=\mathfrak{H} 3 G$. It is easily seen that every element in $\mathfrak{Q} G$ is a $Q-G$-element [Corollary 1.2]. Terming an element $w \in G$ a weak $\mathfrak{Q}$ - $G$-element, if to every $G$-commutator sequence $c_{i}$ with $w=c_{1}$ there exist positive integers $i \neq j$ with $c_{i}=c_{j}$, it is clear that $\mathfrak{Q}-G$ elements are likewis? weak $\mathfrak{Q}-G$-elements. Somewhat deeper is our principal result:

$Q G=$ set of $Q-G$-elements $=$ set of weak $Q-G$-elements [Theorem 3.2].

If $X$ is a group, then we denote by $t X$ the product of all normal torsion subgroups of $X$. This is a characteristic torsion subgroup of $G$ which need not contain all torsion elements of $X$. Then the normal subgroup $N \triangleleft G$ is part of $\mathfrak{Q} G$ if, and only if,

$$
N /(N \cap \mathbb{Q} G) \subseteq \mathfrak{P}[G /(N \cap \mathbb{Q} G)] \text { and } \mathfrak{c}_{\mathfrak{i N}}[(N \cap \mathfrak{Q} G) /(\mathrm{t} N \cap \mathfrak{B} G)] \subseteq \mathfrak{Q} G
$$

where $c_{X} Y$ is the centralizer of $Y$ in $X$ [Theorem 5.2]. In order to prove this and 
related results we derive in section 4 characterizations of the hypercenter which may be of independent interest.

In contrast to the rule $\mathfrak{S}_{3}\left[G / \mathfrak{S}_{3} G\right]=1$ neither of the rules $\mathfrak{P}[G / \mathfrak{P G}]=1$ nor $Q[G / Q G]=1$ is true in general. Thus the following criteria are of interest:

$$
\mathbb{Q}[G / \mathbb{Q} G]=1 \text { if, and only if, } \mathbb{Q}[G / t \mathfrak{Q} G]=1 \text {; }
$$

if $\mathfrak{B} G$ is finite, then $\mathfrak{Q}[G / \mathfrak{B} G]=1$

[Proposition 6.8].

Groups $G$ with $G=\mathfrak{Q} G$ may be termed $\mathfrak{Q}$-groups. Products of finitely many normal $\mathfrak{Q}$-subgroups need not be $\mathfrak{Q}$-groups [Example 7.1], this very much in contrast to hypercentrality. But finitely generated $\mathbb{Q}$-groups are noetherian [Proposition 7.2]. This suggests the definition of locally Q-groups $[=\mathbb{Q Q}$ groups]: these are groups whose finitely generated subgroups are $\mathbb{Q}$-groups. They have the closure property: products of [finitely or infinitely many] normal $\mathfrak{Q Q}$ subgroups are $\mathfrak{E Q}$-groups [ Theorem 7.4].

\section{Notations}

$A \triangleleft B:=: A$ is a normal subgroup of $B$

$A \subset B:=: A$ is a proper subgroup of $B$

$\{\cdots\}=$ subgroup, generated by enclosed subset

$x \circ y=x^{-1} y^{-1} x y=x^{-1} x^{y}$

$x \circ Y=$ set of elements $x \circ y$ with $y \in Y$

$G$-commutator sequence $=$ sequence of elements $c_{i}$ with $c_{i+1} \in c_{l} \circ G$

$c_{G} X=$ centralizer of subset $X$ in $G$

$\mathfrak{C}_{G}(A / B)$ for $B \triangleleft G$ and $B \subseteq A \triangleleft G:=:$ set of all $g \in G$ with $g \circ A \subseteq B$

${ }_{3} G=$ center of $G$

$\mathfrak{H}_{3} G=$ hypercenter of $G$

$\hat{\jmath}_{\sigma} G=\sigma$-th term of ascending central chain of $G$

$G^{\prime}=\{G \circ G\}=$ commutator subgroup of $G$

$\mathfrak{P G}=$ product of finite normal subgroups of $G$

$\mathfrak{Q} G / \mathfrak{P} G=\mathfrak{H}_{3}(G / \mathfrak{P G})$

$o(x)=$ order of [torsion] element $x$

$\mathrm{t} G=$ product of all normal torsion subgroups of $G$

$\mathfrak{I} G=$ set of torsion elements in $G$

Q-group : : : group $X$ with $X=\mathfrak{Q} X$

$\mathfrak{Q} Q$-group $:=:$ group whose finitely generated subgroups are $\mathfrak{Q}$-groups

\section{Basic facts and concepts}

A $G$-Commutator SeQuence [or $G$-commutator series] is a sequence of elements $c_{i} \in G$ [with $0<i$ ] meeting the requirement: 
Here as usual

$$
c_{i+1} \in c_{i} \circ G \text { for } 0<i .
$$

and

$$
x \circ y=x^{-1} y^{-1} x y=x^{-1} x^{y}
$$

$$
x \circ G=\text { set of elements } x \circ g \text { for } g \in G \text {. }
$$

Proposition 1.1. The hypercenter $\mathfrak{S}_{3} G$ is the set of all elements $h \in G$ with the property:

every G-commutator sequence, containing $h$, contains also 1.

TERMINOLOGICAL REMINDERS. $3 G=$ center of $G ; \mathfrak{j} 3 G=$ hypercenter of $G=$ intersection of all $X \triangleleft G$ with $3(G / X)=1$.

This result is a slight generalization of Kuroš [p. 219, Lemma].

Proof. Suppose that $c_{i}$ is a $G$-commutator sequence with $c_{i} \neq 1$ for every $i$. Then there exists a normal subgroup $X \triangleleft G$ with $c_{i} \notin X$ for every $i$ as e.g. $X=1$; and among these there exists a maximal one, say $M$ [Maximum Principle of Set Theory]. Suppose that $3[G / M] \neq 1$. This is equivalent to the existence of $Z$ with

$$
Z \circ G \subseteq M \subset Z \triangleleft G \text {. }
$$

Because of the maximality of $M$ there exists $z$ with $c_{z} \in Z$. Then $c_{z+1} \in c_{z} \circ G \subseteq Z \circ G \subseteq M$, contradicting our choice of $M$. Hence $1=3[G / M]$; and thus it follows from the definition of the hypercenter that $\mathfrak{H} z G \subseteq M$. Since $c_{i} \notin M$ for every $l$, we have a fortiori that $c_{i} \notin \mathfrak{Y}_{3} G$ for every $i$; and we have shown:

(1) If $c_{i}$ is a $G$-commutator sequence with $1 \neq c_{i}$ for every $i$, then $c_{i} \notin \mathfrak{S}_{3} G$ for every $i$.

Consider next an element $g \notin \mathfrak{J}_{3} G$. Then we are going to construct a $G$ commutator sequence $c_{i}$ with $g=c_{1}$ and $c_{i} \notin \mathfrak{S}_{3} G$ for every $i$. To do this we may make the inductive hypothesis that we have already constructed elements $c_{1}, \cdots, c_{n}$ with

$$
g=c_{1}, c_{i} \notin \mathfrak{S}_{3} G \text { for } i \leqq n \text { and } c_{i+1} \in \mathcal{c}_{1} \circ G \text { for } i<n \text {. }
$$

Since in particular $c_{n} \notin \mathfrak{H}_{3} G$, and since [as is easily verified] $1=3\left[G / \mathfrak{J}_{3} G\right]$, the element $c_{n} \mathfrak{H}_{3} G$ does not belong to the center. Hence there exists an element in $G / \mathfrak{S}_{3} G$ which does not commute with $c_{n} \mathfrak{H}_{3} G$; and this implies the existence of $c_{n+1} \in c_{n} \circ G$ with $c_{n+1} \notin \mathfrak{Y}_{3} G$. This completes our inductive argument; and we have shown:

(2) If $g \notin \mathfrak{S}_{3} G$, then there exists a $G$-commutator sequence $c_{i}$ with $g=c_{1}$ and $c_{i} \notin \mathfrak{D}_{3} G$ for every $i$.

Combination of (1) and (2) shows:

(3) The following properties of $g \in G$ are equivalent:

(i) $g \notin \mathfrak{H}_{3} G$. 
(ii) There exists a $G$-commutator sequence which contains $g$, though none of its terms belongs to $\mathfrak{H}_{3} G$.

(iii) There exists a $G$-commutator sequence which contains $g$, but does not contain 1.

Our proposition is equivalent with this statement.

$\mathfrak{B} G=$ product of all finite normal subgroups of $G$.

$\mathbb{Q} G=$ uniquely determined subgroup of $G$ with $\mathfrak{P} G \subseteq \mathbb{Q} G$ and $\mathfrak{Q} G / \mathfrak{P} G=\mathfrak{H}_{3}[G / \mathfrak{B} G]$.

It is clear that $\mathfrak{P G}$ and $\mathfrak{Q} G$ are well determined characteristic subgroups of $G$.

Finitely VALUED is the $G$-commutator sequence $c_{i}$, if only finitely many of the $c_{i}$ are distinct [if the set of the $c_{i}$ is a finite set].

COROLlaRY 1.2. Every G-commutator sequence which contains an element in $Q G$ is finitely valued.

Proof. Assume that the $G$-commutator sequence $c_{i}$ contains an element in

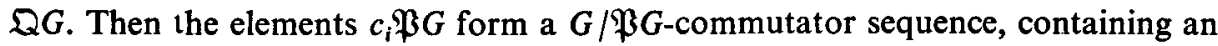
element in $\mathfrak{Q} G / \mathfrak{B} G=\mathfrak{H}_{3}[G / \mathfrak{P} G]$. It is an immediate consequence of Proposition 1.1 that $1=c_{i} \mathfrak{P G}$ for some $i$ [and hence for almost all $i$ ]. Thus some $c_{i}$ belongs to $\mathfrak{P G}$; and this implies the existence of a finite normal subgroup $E \triangleleft G$ which contains some $c_{i}$. Then almost all $c_{i}$ belong to $E$; and we deduce from the finiteness of $E$ that the $G$-commutator sequence $c_{i}$ is finitely valued.

This corollary may also be stated in the following form:

if $g \in \mathfrak{Q} G$, then every $G$-commutator sequence which contains $g$ is finitely valued.

DefinItion 1.3. (A) The element $g \in G$ is a $Q$-G-element, if every G-commutator sequence, containing $g$, is finitely valued.

(B) The element $g \in G$ is a weak Q-G-element, if every G-commutator sequence $g_{i}$ with $g=g_{1}$ satisfies $g_{i}=g_{j}$ for some $i \neq j$.

It is clear that every $\mathbb{Q}$ - $G$-element is a weak $\mathfrak{Q}$ - $G$-element; and it is a consequence of Corollary 1.2 that every element in $\mathfrak{Q} G$ is a $\mathfrak{Q}-G$-element. That conversely every weak $\mathfrak{Q}-G$-element belongs to $\mathfrak{Q} G$, wilı be shown below in Theorem 3.2.

LEMMA 1.4. (A) If $\sigma$ is an epimorphism of $G$ upon $H$, if $h_{i}$ is an H-commutator sequence, and if $g \in G$ with $g^{\sigma}=h_{1}$, then there exists a $G$-commutator sequence $g_{1}$ with $g=g_{1}$ and $g_{i}^{\sigma}=h_{i}$ for every $i$.

(B) Every epimorphism of $G$ upon $H$ maps Q-G-elements upon $Q-H$ elements and weak $\mathfrak{Q}$-G-elements upon weak $\mathfrak{Q}$-H-elements.

Proof. Suppose that $g \in G$, that $\sigma$ is an epimorphism of $G$ upon $H$ and that $h_{i}$ is an $H$-commutator sequence with $g^{\sigma}=h_{1}$. We let $g=g_{1}$; and we make the 
inductive hypothesis that $0<n$ and that elements $g_{1}, \cdots, g_{n}$ have already been constructed, meeting the following requirements:

$$
\begin{aligned}
& g_{i}^{\alpha}=h_{i} \text { fo: } i=1, \cdots, n ; \\
& g_{i+1} \leqslant g_{i} \circ G \text { for } 0<i<n .
\end{aligned}
$$

Since $h_{n+1} \in h_{n} \circ H$, there exists $a \in H$ with $h_{n+1}=h_{n} \circ a$. Since $H=G^{\sigma}$, there exists $b \in G$ with $b^{\sigma}=a$. Let $g_{n+1}=g_{n} \circ b$. Then

$$
\begin{aligned}
& g_{n+1} \in g_{n} \circ G \text { and } \\
& g_{n+1}^{\sigma}=\left(g_{n} \circ b\right)^{\sigma}=g_{n}^{\sigma} \circ b^{\sigma}=h_{n} \circ a=h_{n+1},
\end{aligned}
$$

completing our inductive construction of the desired $G$-commutator sequence $g_{i}$. This proves (A); and (B) is a fairly immediate consequence of (A).

Subgroup inheritance is quite obvious: if $u \in U \subseteq G$, and if $u$ is a $\mathbb{Q}-G$ element [a weak $\mathfrak{Q}-G$-element], then $u$ is a $\mathfrak{Q}-U$-element [a weak $\mathfrak{Q}-U$-element].

LEMMA 1.5. If $g$ is a $\mathbb{Q}$-G-element [a weak $\mathbb{Q}$-G-element], then every element in $g \circ G$ is a $\mathbb{Q}-G$-element [a weak $\mathfrak{Q}-G$-element].

Proof. Suppose that $x \in G$ and that $c_{i}$ is a $G$-commutator sequence with $g \circ x=c_{1}$. Then the sequence $g=c_{0}, c_{1}, c_{2}, \cdots$ is a $G$-commutator sequence too If firstly $g$ is a $Q$-G-element, then the sequence $c$, is finitely valued so that $g \circ x=c_{1}$ is likewise a $\mathbb{Q}-G$-element. Assume next that $c_{i} \neq c_{j}$ for $0<i<j$. If then $g$ were equal to one of the $c_{i}$ with $0<i$, then $g$ would certainly not be a weak $Q$-G-element; and if $g \neq c_{i}$ for $0<i$, then the elements in the sequence $c_{i}$ with $0<i$ would likewise be pairwise different so that again $g$ would not be a weak $\mathbb{Q}$ - $G$-element. Hence $g \circ x$ is a weak $\mathbb{Q}$ - $G$-element whenever $g$ is a weak $\mathbb{Q}-G$ element.

LEMMA 1.6. If $N \triangleleft G$, if $\mathfrak{F}$ is a finite set of normal subgroups of $G$ with $X \subseteq N$ and $N / X \subseteq \mathfrak{S}_{3}(G / X)$ for every $X \in \mathfrak{F}$, then

$$
N / \bigcap_{X \in \mathbb{Z}} X \subseteq \mathfrak{S}_{3}\left[G / \bigcap_{X \in \mathfrak{Z}} X\right] .
$$

Note. The requirement that $\mathfrak{F}$ be finite is indispensable, witness the nonabelian free groups which are certainly not hypercentral, though they are, by a Theorem of Magnus, residually nilpotent; see Specht [11, p. 211, Satz 21].-This result is, presumably, well known; we add the simple proof for the reader's convenience.

Proof. Consider a $G$-commutator sequence $c_{i}$ which contains elements in $N$. If $X \in \mathfrak{F}$, then the $X c_{i}$ form a $G / X$-commutator sequence, containing elements in $N / X \subseteq \mathfrak{S}_{3}(G / X)$. Application of Proposition 1.1 shows that almost all $X c_{i}=1$. Hence almost all $c_{i} \in X$ for every $X \in \mathfrak{F}$. Since $\mathfrak{F}$ is finite, almost all 
Hence

$$
c_{i} \in \bigcap_{X \mathcal{Z}} X
$$

$$
\text { c. } \bigcap_{X \in \mathfrak{Z}} X=1 \text { for almost all } i \text {; }
$$

and now one deduces $N / \bigcap_{X \in \mathfrak{Z}} X \subseteq \mathfrak{S}_{3}\left[G / \bigcap_{X \in \mathfrak{\Im}} X\right]$ from Proposition 1.1 [and Lemma 1.4, (A)].

LEMMA 1.7. If $X, Y, Z$ are normal subgroups of $G$ with $X Y \subseteq Z$ and $Z / X \subseteq \mathfrak{P}(G / X)$, then $Y /(Y \cap X) \subseteq \mathfrak{P}[G /(Y \cap X)]$.

Proof. Clearly

$$
Y /(Y \cap X) \cong Y X / X \subseteq Z / X \subseteq \mathfrak{P}(G / X) .
$$

Thus $Y X / X$ is a product of finite normal subgroups of $G / X$. Since the isomorphism $Y X / X \cong Y /(Y \cap X)$ is a $G$-isomorphism, we deduce that $Y /(Y \cap X)$ is a product of finite normal subgroups of $G /(Y \cap X)$; and this implies $Y /(Y \cap X) \subseteq \mathfrak{P}[G /(Y \cap X)]$.

\section{Automorphisms of torsionfree abelian groups}

We are going to discuss in this section torsion automorphisms of torsionfree abelian groups which meet a requirement analogous to the $\mathfrak{Q}$-property of group elements.

(2.1) The multiplicative order of the complex root of unity $e$ is 6 if, and only if, $1-e$ is a root of unity.

Proof. Let $e=a+i b$ with real $a, b$ and $i=(-1)^{\frac{1}{2}}$. Then $e$ and $1-e$ have both absolute value 1 if, and only if,

$$
a^{2}+b^{2}=1 \text { and }(1-a)^{2}+(-b)^{2}=1 \text {. }
$$

This implies $a=\frac{1}{2}$ and $b^{2}=\frac{3}{4}$; and (2.1) is a fairly immediate consequence of this.

It will be convenient to denote the composition of the abelian groups under consideration in this section 2 as addition $a+b$; and the effect of the endomorphism $\beta$ on the element $a$ will be designated by $a \beta$.

(2.2) If $\beta$ is a torsion automorphism of the free abelian group $F$ of finite rank, if $F / U$ is finite for every $\beta$-admissible subgroup $U \neq 0$ of $F$, if the order $o(\beta) \neq 6$, and if there exists $0 \neq a \in F$ and integers $i, j$ with $0 \leqq i<j$ and $a(1-\beta)^{i}=a(1-\beta)^{j}$, then $\beta=1$.

Proof. Denote by $\theta$ the ring of endomorphisms of $F$ which is spanned by $\beta$. Naturally $\theta$ is commutative. If $0 \neq \sigma \in \theta$, then $F \sigma$ is an infinite free abelian group 
so that the kernel of $\sigma$ is not of finite index in $F$. But the kernel of $\sigma$ is $\beta$-admissible because of the commutativity of $\theta$ [and $\beta, \sigma \in \theta$ ]. It follows therefore from our hypothesis that the kernel of $\sigma$ is 0 ; and we have shown:

(1) If $f \in F$ and $\sigma \in \theta$ with $f \sigma=0$, then $f=0$ or $\sigma=0$.

Application of Baer [3; p. 143, Folgerung 1] shows furthermore:

(2) $\theta$ is a domain of integrity and $\theta_{+}$is torsionfree of finite rank so that $\theta$ is a subring of a finite algebraic number field.

[This could also be directly deduced from (1).]

Assume by way of contradiction that $\beta \neq 1$. There exist by hypothesis $0 \neq a \in F$ and integers $i, j$ with $0 \leqq i<j$ and

$$
a(1-\beta)^{i}=a(1-\beta)^{j} .
$$

Because of (1) and $a \neq 0$ it follows that

$$
0=(1-\beta)^{i}-(1-\beta)^{j} ;
$$

and from $1-\beta \neq 0$ and (2) together with $0<j-i$ we deduce

$$
1=(1-\beta)^{j-i} \text {. }
$$

Thus $\beta$ and $1-\beta$ are [by (2)] complex roots of unity; and this implies $o(\beta)=6$ because of $(2.1)$, a contradiction proving the desired $\beta=1$.

Remark 2.3. Denote by $e$ a primitive [complex] 6-th root of unity and let $R$ be the ring of complex numbers, spanned by $e$. Then $R$ is a domain of integrity and $F=R_{+}$is a free abelian group of finite rank. It is a consequence of (2.1) that $1-e$ too is a root of unity.

If $\beta$ is the automorphism of $F$, effected by multiplication by $e$, then all the hypotheses of (2.2) are satisfied by $F$ and $\beta$ with the exception of $o(\beta) \neq 6$. This shows the indispensability of this hypothesis.

(2.4) If $\beta$ is a torsion automorphism of the torsionfree abelian group $F \neq 0$, if $o(\beta)$ is not a multiple of 6 , and if for every $0 \neq a \in F$ there exist integers $i, j$ with $0 \leqq i<j$ and $a(1-\beta)^{i}=a(1-\beta)^{j}$, then there exists $0 \neq f \in F$ with $f=f \beta$.

Proof. If $f \in F$, then the set $f, f \beta, \cdots, f \beta^{o(\beta)-1}$ is $\beta$-invariant so that the finitely generated subgroup $\left\{f, f \beta, \cdots, f \beta^{o(\beta)-1}\right\}$ is $\beta$-admissible. This subgroup is free abelian of finite rank, since $F$ is torsionfree. Hence

(1) every element in $F$ is contained in a $\beta$-admissible subgroup of $F$ which is free abelian of finite rank.

From $F \neq 0$ and (1) it follows that there exist $\beta$-admissible subgroups of $F$ which are free abelian of positive rank; and among these there exists one, say $A$, of minimal rank. Then $A \neq 0$ is free abelian of finite rank, $A=A \beta$. 
$0 \subset B=B \beta \subseteq A$, then $A$ and $B$ have the same rank because of the minimality of the rank of $A$; and this is equivalent with the finiteness of $A / B$. We have shown:

(2) there exists a $\beta$-admissible free abelian subgroup $A \neq 0$ of finite rank of $F$ such that $0 \subset B=B \beta \subseteq A$ implies the finiteness of $A / B$.

Denote by $\tilde{\beta}$ the automorphism, induced by $\beta$ in $A$. Then $o(\tilde{\beta})$ is a divisor of $o(\beta)$; and $o(\tilde{\beta}) \neq 6$, since $o(\beta)$ is by hypothesis not a multiple of 6 . If $a \in A$, then $a(1-\bar{\beta})^{i}=a(1-\beta)^{i}$; and it follows from our hypothesis that there exist integers $i, j$ with $0 \leqq i<j$ and

$$
a(1-\tilde{\beta})^{i}=a(1-\beta)^{i}=a(1-\beta)^{j}=a(1-\tilde{\beta})^{j} .
$$

Consequently we may apply (2.2) on the torsion automorphism $\tilde{\beta}$ of $A$. Hence $\tilde{\beta}=1$, proving that every element in $A \neq 0$ is a fixed element of $\beta$.

(2.5) If $\beta$ is a torsion automorphism of the torsionfree abelian group $F$, if $o(\beta)$ is not a multiple of 6 , and if to every $0 \neq a \in F$ there exist integers $i, j$ with $0 \leqq i<j$ and $a(1-\beta)^{i}=a(1-\beta)^{j}$, then $\beta=1$.

Proof. Denote by $V$ the set of all $v \in F$ with $v \beta=v$. This is a $\beta$-admissible subgroup of $F$. Assume by way of contradiction that $V \subset F$. Then $F^{*}=F / V \neq 0$ and $\beta$ induces in $F^{*}$ a torsion automorphism $\beta^{*}$. Since $o(\beta)$ is a multiple of $o\left(\beta^{*}\right)$, and since $o(\beta)$ is not a multiple of $6, o\left(\beta^{*}\right)$ is not a multiple of 6 .

If $a \in F$, then $a^{*}=a+V \in F^{*}$ and

$$
a^{*}\left(1-\beta^{*}\right)^{i}=a(1-\beta)^{i}+V .
$$

If $a \neq 0$, then there exist by hypothesis integers $i, j$ with $0 \leqq i<j$ and $a(1-\beta)^{i}=a(1-\beta)^{j}$. It follows that

$$
a^{*}\left(1-\beta^{*}\right)^{i}=a(1-\beta)^{i}+V=a(1-\beta)^{j}+V=a^{*}\left(1-\beta^{*}\right)^{j} .
$$

Consequently we may apply (2.4) on $F^{*}$ : there exists an element $w^{*} \neq 0$ in $F^{*}$ with $w^{*}=w^{*} \beta^{*}$.

Naturally there exists an element $w \in F$ with $w^{*}=w+V$; and this element $w \in F$ has the following properties:

$$
w \notin V \text { and } w(\beta-1) \in V .
$$

In particular $w(\beta-1) \neq 0$, since otherwise $w$ would be a fixed element of $\beta$ and would as such belong to $V$. From

$$
w \beta=w+w(\beta-1)
$$

and $w(\beta-1) \beta=w(\beta-1)$ because of $w(\beta-1) \in V$, we deduce by complete induction

$$
w \beta^{i}=w+i w(\beta-1) \text { for every positive integer } i .
$$

This implies in particular that 


$$
w=w \beta^{o(\beta)}=w+o(\beta) w(\beta-1) .
$$

Hence $o(\beta) w(\beta-1)=0$; and this implies the contradiction $w(\beta-1)=0$, since $F$ is torsionfree. From this contradiction we deduce that $F=V$; and this implies $\beta=1$.

LEMMA 2.6. If $\theta$ is a finite group of automorphisms of the torsionfree abelian group $F$, then the element $a \in F$ is a fixed element of $\theta$, if (and only if) there exist to every $\rho \in \theta$ integers $i, j$ with $0 \leqq i<j$ and $a(1-\rho)^{i}=a(1-\rho)^{j}$.

Proof. $A=\{a \theta\}$ is a finitely generated subgroup of $F$, since $\theta$ is finite; and hence $A$ is free abelian of finite rank. Naturally $A$ is $\theta$-admissible so that $\theta$ induces in $A$ a finite group $\wedge$ of automorphisms. If $\wedge \neq 1$, then $\wedge$ contains an automorphism $\lambda$ of $A$ with $o(\lambda)$ a prime $p$. Then $\lambda \neq 1$, so that the exists $\omega \in \theta$ with $a \omega \lambda \neq a \omega$. Then $\omega$ induces in $A$ an automorphism $\tilde{\omega}$ and $\tilde{\omega} \in \wedge$ so that $\tilde{\omega} \lambda \tilde{\omega}^{-1}=\beta$ is an automorphism in $\wedge$ with $o(\beta)=p$ and $a \beta \neq a$. We have shown:

(1) If $\wedge \neq 1$, then there exists $\beta \in \wedge$ with $o(\beta)=p$, a prime, and $a \beta \neq a$.

Let $B=\left\{a, a \beta, a \beta^{2}, \cdots, a \beta^{p-1}\right\}$. This is a $\beta$-admissible subgroup of $A$ and $F$ and as such $B$ is a free abelian group of finite rank. Note that $a \neq 0$ because of $a \neq a \beta$. There exists $\rho \in \theta$, inducing $\beta$ in $A$. By hypothesis, there exist integers $i, j$ with $0 \leqq i<j$ and $a(1-\rho)^{i}=a(1-\rho)^{j}$. Since $a(1-\rho)^{n}=a(1-\beta)^{n}$, we have shown:

(2) There exist integers $i, j$ with $0 \leqq i<j$ and $a(1-\beta)^{i}=a(1-\beta)^{j}$.

If $0<k<p$, then

$$
\left[a \beta^{k}\right](1-\beta)^{i}=\left[a(1-\beta)^{i}\right] \beta^{k}=\left[a(1-\beta)^{j}\right] \beta^{k}=\left[a \beta^{k}\right](1-\beta)^{j} .
$$

If $c_{k}$ is for $k=0, \cdots, p-1$ an integer, then

$$
\begin{aligned}
{\left[\sum_{k=0}^{p-1} c_{k} a \beta^{k}\right](1-\beta)^{i} } & =\sum_{k=0}^{p-1} c_{k}\left[a \beta^{k}(1-\beta)^{i}\right] \\
& =\sum_{k=0}^{p-1} c_{k}\left[a \beta^{k}(1-\beta)^{j}\right]=\left[\sum_{k=0}^{p-1} c_{k} a \beta^{k}\right](1-\beta)^{j}
\end{aligned}
$$

Thus it follows from (2) that

(3) there exist integers $i, j$ with $0 \leqq i<j$ and $b(1-\beta)^{i}=b(1-) \beta^{j}$ for every $b \in B$.

Since $B$ is $\beta$-admissible, an automorphism $\beta^{*}$ is induced by $\beta$ in $B$. Since $a \beta \neq a, \beta^{*} \neq 1$ and since $o(\beta)=p$ a prime, it follows that $o\left(\beta^{*}\right)=p$. Since $o\left(\beta^{*}\right)$ is a prime, $o\left(\beta^{*}\right)$ is not a multiple of 6 . By (3) we may apply (2.5) to show that $\beta^{*}=1$, the desired contradiction.

We have shown that $\Lambda=1$ and that therefore $\theta$ fixes every element in $A$. Thus in particular $a=a \theta$, as we wanted to show. 
COROLLARY 2.7. Suppose that $\theta$ is a group of automorphisms of the torsionfree abelian group $F$ and that $F$ is generated by its elements $f$ with the following two properties:

(a) $f \theta$ is finite;

(b) to every $\rho \in \theta$ there exist integers $i, j$ with $0 \leqq i<j$ and $f(1-\rho)^{i}=f(1-\rho)^{j}$.

Then $\theta=1$.

This is an immediate consequence of Lemma 2.6.

\section{The main theorem}

TERMINOLOGICAL ReMINDER. $\mathfrak{I} X=$ set of torsion elements in the group $X$.

Note that $\mathfrak{I} X$ need not be a subgroup of $X$; if $\mathfrak{I} X$ happens to be a subgroup of $X$, then it is a characteristic torsion subgroup of $X$.

(3.0) (A) If $X$ is a hypercentral group, then $\mathfrak{I} X$ is a locally finite characteristic subgroup of $X$.

(B) $\mathfrak{I Q X}$ is always a locally finite characteristic subgroup of $X$ with $\mathfrak{I Q X} / \mathfrak{P} X=\mathfrak{I}\left[\mathfrak{H}_{3}(X / \mathfrak{P} X)\right]$.

Proof. (A) is a well known fact; see Baer [1; p. 207, Corollary].

Since $\mathfrak{S}_{3} X$ is always hypercentral, it follows that $\mathfrak{I S}_{3} X$ is always a locally finite characteristic subgroup. Likewise $\mathfrak{B X}$ is always a locally finite characteristic subgroup. Finally extensions of locally finite groups by locally finite groups are locally finite; see e.g. Specht [p. 141, Satz $40^{*}$ ]. Combine these three facts to obtain (B).

TERMinOLOGICAL REMINDER. If $x$ and $y$ are elements in $G$, the elements $x \circ^{(n)} y$ are inductively defined by the rules:

$$
x \circ^{(0)} y=x, x \circ^{(n+1)} y=\left(x \circ^{(n)} y\right) \circ y .
$$

This series of elements is clearly a $G$-commutator sequence, beginning with $x$.

Lemma 3.1. If $e \in G$ and the set $e^{G}$ of elements, conjugate to $e$ in $G$, is finite, then the normal subgroup $E=\left\{e^{G}\right\} \triangleleft G$ has the following properties:

$$
\left\{\begin{array}{l}
\text { (a) } G / \mathfrak{c}_{G} E \text { and } E / 3 E \text { are finite. } \\
\text { (b) } E^{\prime} \text { and } \mathfrak{I} E \text { are finite normal subgroups of } G . \\
\text { (c) } E / \mathfrak{T} E \text { is free abelian of finite rank. }
\end{array}\right.
$$

(B) If to every $x \in G$ there exist integers $i, j$ w:th $0 \leqq i<j$ and $e \circ^{(i)} x=e \circ^{(j)} x$, then $E \circ G \subseteq \mathfrak{I} E$.

(C) If e is a weak $\mathfrak{Q}$-G-element, then $E \circ G \subseteq \mathfrak{I} E$. 
Proof. It is clear that an element in $G$ centralizes $E$ if, and only if, it centralizes every element in $e^{G}$. Furthermore every element in $G$ induces a permutation of the set $e^{\sigma}$. It follows that $G / c_{G} E$ is essentially the same as the group of permutations, induced by $G$ in the set $e^{G}$. Since $e^{G}$ is finite by hypothesis, $G / \mathfrak{c}_{G} E$ is finite. Hence

$$
E / 3 E=E /\left(E \cap \mathfrak{c}_{G} E\right) \cong E \mathfrak{c}_{G} E / \mathfrak{c}_{G} E \subseteq G / \mathfrak{c}_{G} E
$$

is likewise finite: we have verified (A.a).

From the finiteness of $E / 3 E$ and a Theorem of I. Schur we deduce the finiteness of $E^{\prime}$; see Huppert [p. 417, 2.3 Satz]. Since $E$ is finitely generated, so is the abelian group $E / E^{\prime}$. Hence $\mathfrak{I}\left(E / E^{\prime}\right)$ is a finite group and $\left(E / E^{\prime}\right) / \mathfrak{I}\left(E / E^{\prime}\right)$ is a free abelian group of finite rank. Consequently

$$
\mathfrak{I}\left(E / E^{\prime}\right)=\mathfrak{T} E / E^{\prime} \text { and }\left(E / E^{\prime}\right) / \mathfrak{I}\left(E / E^{\prime}\right) \cong E / \mathfrak{I} E \text {, }
$$

since $E^{\prime}$ is finite, completing the proof of $(A \cdot b+c)$.

Assume now that $e$ meets the following requirement:

(0) To every $x \in G$.here exist integers $i, j$ with $0 \leqq i<j$ and

$$
e{ }^{(i)} x=e o^{(i)} x
$$

Let $X^{*}=X \mathfrak{T} E / \mathfrak{I} E$ for every subset $X$ of $G$. Then $E^{*}=\left\{e^{* \mathrm{G}^{*}}\right\} \triangleleft G^{*}$ is free abelian of finite rank by (A.c).

Since $G / \mathfrak{c}_{G} E$ is finite by (A.a), and since $\mathfrak{c}_{G} E \mathfrak{T} E / \mathfrak{T} E \subseteq \mathfrak{c}_{G^{*}} E^{*}$, it follows that $G^{*} / c_{G} E^{*}$ is finite. But this latter group is essentially the same as the group $\theta$ of automorphisms, induced in $E^{*}$ by $G^{*}$; and thus we have shown:

(1) $E^{*} \triangleleft G^{*} ; E^{*}$ is free abelian of finite rank; the group $\theta$ of automorphisms induced in $E^{*}$ by $G^{*}$, is finite.

If $\beta \in \theta$, then $\beta$ is induced in $E^{*}$ by an element $b \in G^{*}$. If $x \in E^{*}$, then

$$
x \circ b=x^{-1} x^{\beta}=x^{-1} x^{\beta}=x^{\beta-1} .
$$

From (2.a) one deduces by complete induction that

$$
x \circ^{(i)} b=x^{(\beta-1)^{t}} \text { for } i=0,1,2, \cdots .
$$

Hence it follows from our hypothesis $(0)$ that

(2.c) there exist integers $i, j$ wi.h $0 \leqq i<j$ and $e^{*(\beta-1)^{t}}=e^{*(\beta-1)^{\prime}}$.

Since $e^{*}, e^{* \beta}, e^{* \beta-1}$ belong to the abelian group $E^{*}$, it follows that

$$
e^{*(1-\beta)^{2 i}}=e^{*(\beta-1)^{2 t}}=e^{*(\beta-1)^{2 j}}=e^{*(1-\beta)^{2 j}} .
$$

Thus we have shown that

(2) to every $\rho \in \theta$ there exist integers $h, k$ with $0 \leqq h<k$ and

$$
e^{*(1-\rho)^{h}}=e^{*(1-\rho)^{k}} \text {. }
$$


Because of (1) and (2) we may apply Lemma 2.6 on $e^{*} \in E^{*}$ and the finite group $\theta$ of automorphisms of $E^{*}$ :

$$
e^{*} \text { is a fixed element of } \theta \text {. }
$$

But this is equivalent with $e^{*} \circ G^{*}=1$; and this is in turn equivalent with

$$
e \circ G \subseteq \mathfrak{I} E \text {. }
$$

If $g \in G$, then we deduce from (3) that

$$
e^{g} \circ G \subseteq \mathfrak{I} E,
$$

since $\mathfrak{T} E \triangleleft G$. From $E=\left\{e^{G}\right\}$ it follows therefore that $E \circ G \subseteq \mathfrak{T} E$, proving (B).

That $(C)$ is a consequence of $(B)$, is an immediate consequence of the fact that the elements $e{ }^{(i)} x$ form for every $x \in G$ a $G$-commutator sequence, beginning with $e$.

THEOREM 3.2. $Q G=$ set of all $Q-G-e l e m e n t s=$ set of all weak $Q-G-$ elements.

Proof. It is a consequence of Corollary 1.2 that every element in $Q G$ is a $\mathbb{Q}$ - $G$-element; and it is obvious-see Definition 1.3-that every $\mathbb{Q}-G$-element is a weak $Q$-G-element. Hence all we have to prove is the fact that every weak $Q-G$ element belongs to $Q G$.

Consider an element $g \in G$ such that $g \circ G$ is a subset of $Q G$. Then $(g \mathfrak{P} G) \circ(G / \mathfrak{P G})$ is a subset of $\mathfrak{Q} G / \mathfrak{P} G=\mathfrak{H}_{3}(G / \mathfrak{P} G)$. Hence

$$
[g \mathfrak{P}] \mathfrak{S}_{3}(G / \mathfrak{P} G) \in \mathfrak{3}\left[(G / \mathfrak{P} G) / \mathfrak{H}_{3}(G / \mathfrak{P} G)\right]=1 \text {. }
$$

Thus we have shown that $g \mathfrak{P G} \in \mathfrak{H}_{3}(G / \mathfrak{P G})=\mathfrak{Q} G / \mathfrak{P G}$; and this proves:

(1) If $g \in G$ and $g \circ G$ is a subset of $\mathbb{Q} G$, then $g \in \mathbb{Q} G$.

Consider a weak $\mathfrak{Q}-G$-element $e$ with finite $e^{G}$. Let $E=\left\{e^{G}\right\}$. Then $E \triangleleft G$ and it follows from Lemma 3.1, (A.b) + (C) that

$\mathfrak{I} E$ is a finite normal subgroup of $G$ and

$$
e \circ G \subseteq E \circ G \subseteq \mathfrak{I} E \subseteq \mathfrak{H} G \subseteq \mathfrak{Q} G \text {. }
$$

Application of (1) shows that $e \in \mathbb{Q} G$, proving:

(2) If $e$ is a weak $\mathbb{Q}$ - $G$-element with finite $e^{G}$, then $e \in \mathbb{Q} G$.

Assume that $e$ is a weak $\mathfrak{Q}-G$-element with the property that almost all elements in $e \circ G$ belong to $\mathbb{Q} G$ and that $e \notin \mathbb{Q} G$. If $e \circ G$ were a subset of $\mathbb{Q} G$, then we would deduce $e \in \mathbb{Q} G$ from (1), contradicting our assumptions. Hence there exists $w \in G$ with $e \circ w \notin \mathbb{Q} G$.

If $e^{G}$ were finite, then we would deduce $e \in \mathbb{Q} G$ from (2), contradicting our hypothesis. Hence 


$$
e^{G} \text { is infinite. }
$$

Because of $e \circ x=e^{-1} e^{x}$ the set $e \circ G$ is, by (3.b), infinite. Since almost all the elements in $e \circ G$ belong to $Q G$, it follows from the infinity of $e \circ G$ that an infinity of elements in $e \circ G$ belongs to $Q G$. In other words:

$(e \circ G) \cap \mathbb{Q} G$ is an infinite set.

Since $\mathfrak{Q} G$ is a characteristic subgroup, $(e \circ G)^{w} \cap \mathbb{Q} G$ is likewise an infinite subset of $\mathbb{Q} G$; and hence it follows from (3.a) that

(3.d) $\quad(e \circ w)\left[(e \circ G)^{w} \cap \mathbb{Q} G\right]$ is an infinite set of elements none of which belongs to $\mathbb{Q} G$.

Next we note that by Huppert [p. 253, 1.2 Hilfssatz $b$ )]

Thus

$$
(e \circ w)(e \circ x)^{w}=e \circ x w \in e \circ G \text { for every } x \in G \text {. }
$$

$$
(e \circ w)\left[(e \circ G)^{w} \cap Q G\right] \text { is a subset of } e \circ G \text {; }
$$

and it follows from (3.d) that this is an infinite subset of $e \circ G$ none of whose elements belongs to $\mathbb{Q} G$. This contradicts our assumption that almost all elements in $e \circ G$ belong to $\mathbb{Q} G$; and this contradiction shows:

(3) If $e$ is a weak $Q-G$-element and if almost all elements in $e \circ G$ belong to $\mathbb{Q} G$, then $e \in \mathbb{Q} G$.

Consider a weak $\mathfrak{Q}-G$-element $e$ and assume by way of contradiction that $e \notin Q G$. We let $e=c_{1}$; and we assume that $0<n$ and that we have already constructed elements $c_{1}, \cdots, c_{n}$ with the following properties:

$$
\begin{aligned}
& c_{i} \notin \mathbb{Q} G \text { for } i=1, \cdots, n ; \\
& c_{i} \neq c_{j} \text { for } 1 \leqq i<j \leqq n ; \\
& c_{i+1} \in c_{\imath} \circ G \text { for } 0<i<n .
\end{aligned}
$$

Since $c_{1}=e$ is a weak $\mathfrak{Q}-G$-element, so is, by Lemma 1.5 , every $c_{i}$. In particular $c_{n}$ is a weak $\mathbb{Q}$-G-element which by (4.a) does not belong to $\mathfrak{Q} G$. It is therefore a consequence of (3) that infinitely many elements in $c_{n} \circ G$ do not belong to $Q G$. Consequently there exists $c_{n+1} \in c_{n} \circ G$ with $c_{n+1} \notin \mathbb{Q} G$ and $c_{n+1} \neq c_{i}$ for $i=1, \cdots, n$. This completes the inductive construction of a $G$-commutator series $c_{i}$ with $e=c_{1}\left[c_{i} \notin \mathbb{Q} G\right.$ for every $\left.i\right]$ and $c_{i} \neq c_{j}$ for $i \neq j$. This contradicts the fact that $e$ is a weak $\mathfrak{Q}-G$-element; and this contradiction shows that $e \in \mathbb{Q} G$. Hence

$$
\text { every weak } \mathfrak{Q} \text { - } G \text {-e.ement belongs to } \mathfrak{Q} G \text {, }
$$

as we intended to prove.

COROLLARY 3.3. If $N \triangleleft G$, then 
(a) $[N /(N \cap \mathfrak{B G})] \cap \mathfrak{S}_{3}[G /(N \cap \mathfrak{P G})]=(N \cap \mathbb{Q} G) /(N \cap \mathfrak{B G})$;

(b) $[N /(N \cap \mathfrak{P G})] /\left([N /(N \cap \mathfrak{P G})] \cap \mathfrak{W}_{3}[G /(N \cap \mathfrak{P} G)]\right) \cong N /(N \cap \mathbb{Q} G)$;

(c) $[G /(N \cap \mathfrak{P} G)] /\left([N /(N \cap \mathfrak{P} G)] \cap \mathfrak{H}_{3}[G /(N \cap \mathfrak{P} G)]\right) \cong G /(N \cap \mathfrak{Q} G)$.

Proof. Consider an element $x \in N$. Then it follows from Proposition 1.1 that $(N \cap \mathfrak{P} G) x$ belongs to $\mathfrak{h}_{3}[G /(N \cap \mathfrak{B} G)]$ if, and only if, every $G /(N \cap \mathfrak{P} G)$ commutator sequence, containing $(N \cap \mathfrak{B} G) x$, likewise contains 1 ; and this is by Lemma 1.4, (A) equivalent with the fact that every $G$-commutator sequence, containing $x$, contains elements in $N \cap \mathfrak{B} G$. Note that every element in $\mathfrak{B G}$ is contained in a finite normal subgroup of $G$. Hence our last property is equivalent with the property that every $G$-commutator sequence, containing $x$, is finitely valued; and this is, by definition, the same as saying that $x$ is a $\mathfrak{Q}$-element. Apply Theorem 3.2 to see that this is equiva.ent with $x \in \mathfrak{Q} G$. Thus we have shown:

$x \in N \cap \mathfrak{Q} G$ if, and only if, $(N \cap \mathfrak{P G}) x$ belongs to

$$
[N /(N \cap \mathfrak{B})] \cap \mathfrak{H}_{3}[G /(N \cap \mathfrak{B G})] \text {. }
$$

This fact is essentially the same as our equation (a); and the isomorphisms (b) and (c) are readily deduced from (a).

\section{The hypercentrally imbedded normal subgroups}

The criteria for a normal subgroup to be part of the hypercenter which we are going to derive in this section will be fundamental for the derivation of criteria for a normal subgroup to be contained in the $Q$-subgroup which will be the object of section 5 .

Proposition 4.0. The following properties of $N \triangleleft G$ are equivalent:

(i) $N$ is torsionfree and $N \subseteq \mathfrak{H}_{3} G$.

(ii) $N \cap \mathfrak{P G}=1$ and $N /\left(N \cap \mathfrak{H}_{3} G\right) \subseteq \mathfrak{P}\left[G /\left(N \cap \mathfrak{G}_{3} G\right)\right]$.

We precede the proof proper of this result by the derivation of various properties some of which are not contained in this proposition.

LEMMA 4.1. If $N \sqsupset G$ with $N \cap \mathfrak{P G}=1$ and finite $N /(N \cap 3 G)$, then $N$ is torsionfree with $N \subseteq{ }_{3} G$.

Proof. Every torsion element in $N \cap_{3} G$ generates a finite normal subgroup of $G$ and belongs therefore to $N \cap \Re G=1$. Hence

$N \cap_{3} G$ is torsionfree.

Since $N /\left(N \cap{ }_{3} G\right)$ is finite, $N / 3 N$ is finite too. Application of a Theorem of $\mathrm{I}$. Schur shows therefore the finiteness of $N^{\prime}$; see Huppert [p. 4.17, 2.3 Satz]. 
From $N \triangleleft G$ it follows that $N^{\prime}$ is a finite normal subgroup of $G$. Hence $N^{\prime} \subseteq N \cap \mathfrak{B G}=1$, proving the commutativity of $N$. Ii follows that $\mathfrak{I} N$ is a characteristic torsion subgroup of $N$, implying $\mathfrak{I} N \triangleleft G$ and, by (1),

$$
\mathfrak{I} N \cap\left(N \cap{ }_{3} G\right)=1 \text {. }
$$

From the finiteness of $N /\left(N \cap \cap_{3} G\right)$ we deduce now the finiteness of $\mathfrak{I} N$. Hence $\mathfrak{I} \subseteq N \cap \mathfrak{P G}=1$; and we have shown that

$$
N \text { is a torsionfree abelian group. }
$$

If $x \in N$, then we deduce from the finiteness of $N /\left(N \cap_{3} G\right)$ the existence of a positive integer $n$ with $x^{n} \in N \cap z G$. If $g \in G$, then $x, x^{g}$ and $x \circ g$ belong to the torsionfree abelian normal subgroup $N \triangleleft G$. Hence

$$
(x \circ g)^{n}=\left(x^{-1} x^{g}\right)^{n}=x^{-n} x^{g n}=x^{-n} x^{n g}=x^{-n} x^{n}=1,
$$

since $x^{n} \in\{G$; and this implies $x \circ g=1$, since $N$ is, by (2), torsionfree. Thus we have shown $N \circ G=1$, implying

$$
N \subseteq z G \text {. }
$$

The statements (2) and (3) show the validity of our lemma.

LEMMA 4.2. If $N$ is a torsionfree normal subgroup of $G$ with $N \subseteq \mathfrak{S}_{3} G$, and if $g \in G$ induces a torsion automorphism in $N$, then $g \circ N=1$.

Proof. If this were false, then there would exist $y \in N$ with $y \circ g \neq 1$. Define inductively the commutator sequence $y 0^{(i)} g$ by the rules:

$$
y \circ^{(0)} g=y, y \circ^{(i+1)} g=\left[y \circ^{(i)} g\right] \circ g .
$$

Then $y \circ^{(1)} g \neq 1$; and $y \circ^{(i)} g=1$ for almost all $i$ by Proposition 1.1, since $y \in N \subseteq \mathfrak{H}_{3} G$. Consequently there exists an integer $k$ with

$$
y \circ^{(k-1)} g \neq 1=y \circ^{(k)} g \text {; }
$$

and clearly $2 \leqq k$. Thus $z=y \circ^{(k-2)} g$ is a well determined element in $N$ with the following properties:

$$
z \circ g \neq 1,(z \circ g) \circ g=1 ;
$$

and this implies $(z \circ g)^{g}=z \circ g$. Since

$$
z^{g}=z(z \circ g)
$$

one may prove by complete induction that

$$
z^{\mathbf{g}^{i}}=z(z \circ g)^{i} \text { for every positive integer } i \text {. }
$$

Since $g$ induces a torsion automorphism in $N$, there exists a positive integer $n$ such that 


$$
z=z^{q^{n}}=z(z \circ g)^{n} .
$$

Hence $1=(z \circ g)^{n}$, though $z \circ g \neq 1$. This contradicts the fact that $z \circ g$ is an element in the torsionfree group $N$, a contradiction proving our claim.

Lemma 4.3. If $N \triangleleft G$ with finite $N /\left(N \cap \mathfrak{S}_{3} G\right)$ and $N \cap \mathfrak{P G}=1$, then $\mathfrak{c}_{N}\left(N \cap \mathfrak{H}_{3} G\right)$ is an abelian normal subgroup of $G$ and $N$ is torsionfree with $N \subseteq \mathfrak{S}_{3} G$.

Proof. Since the hypercenter is always a hypercentral group, the set $\mathfrak{I F}_{3} G$ is a characteristic subgroup; cp. Baer [1; p. 207, Corollary]. Consequently

$$
\mathfrak{I}\left[N \cap \mathfrak{S}_{3} G\right]=N \cap \mathfrak{I S}_{3} G \triangleleft G .
$$

If this normal subgroup of $G$ which is part of $\mathfrak{H}_{3} G$ were not 1 , then $3 G \cap \mathfrak{I}\left[N \cap \mathfrak{S}_{3} G\right] \neq 1$. But every torsion element in the center generates a finite normal subgroup so that

$$
1 \subset{ }_{3} G \cap \mathfrak{I}\left[N \cap \mathfrak{S}_{3} G\right] \subseteq N \cap \mathfrak{B G}=1
$$

by hypothesis; and this contradiction shows that $\mathfrak{I}\left[N \cap \mathfrak{G}_{3} G\right]=1$. Thus we have shown that

$$
N \cap \mathfrak{S}_{3} G \text { is torsionfree. }
$$

It is clear tha.

$$
A=\mathfrak{c}_{N}\left(N \cap \mathfrak{S}_{3} G\right)=N \cap \mathfrak{c}_{G}\left(N \cap \mathfrak{S}_{3} G\right) \triangleleft G .
$$

Furthermore

$$
A \cap \mathfrak{S}_{3} G=\left(N \cap \mathfrak{S}_{3} G\right) \cap \mathfrak{C}_{G}\left(N \cap \mathfrak{S}_{3} G\right)=3\left(N \cap \mathfrak{H}_{3} G\right) \subseteq 3_{3} A,
$$

implying

$$
A \cap \mathfrak{S}_{3} G \subseteq \mathfrak{3}_{3} A .
$$

Finally

$A /\left(A \cap \mathfrak{S}_{3} G\right)=A /\left[A \cap\left(N \cap \mathfrak{S}_{3} G\right)\right] \cong A\left(N \cap \mathfrak{Y}_{3} G\right) /\left(N \cap H_{3} G\right) \subseteq N /\left(N \cap \mathfrak{H}_{3} G\right)$ is finite by hypothesis. Combine this with (2.b) to see that

$A / 3 A$ is finite.

Thus we may apply a Theorem of I. Schur to see that $A^{\prime}$ is finite; see Huppert [p. 417, 2.3 Satz]. Since $A^{\prime} \triangleleft G$ is a consequence of $A \triangleleft G$ [by (2.a)], it follows that

$$
A^{\prime} \subseteq N \cap \mathfrak{P G}=1
$$

by hypothesis proving that 


$$
\mathfrak{c}_{N}\left(N \cap \mathfrak{H}_{3} G\right)=A \text { is an abelian normal subgroup of } G \text {. }
$$

Consequently $\mathfrak{I} A$ is a characteristic subgroup of $A$ and as such $\mathfrak{I} A \triangleleft G$. It is a consequence of $\left(1\right.$, that $\mathfrak{I} A \cap\left(N \cap \mathfrak{S}_{3} G\right)=1$. Hence

$$
\mathfrak{I} A=\mathfrak{I} A /\left[\mathfrak{I} A \cap\left(N \cap \mathfrak{S}_{3} G\right)\right] \cong\left(N \cap \mathfrak{S}_{3} G\right) \mathfrak{I} A /\left(N \cap \mathfrak{H}_{3} G\right) \subseteq N /\left(N \cap \mathfrak{S}_{3} G\right)
$$

is finite by hypothesis. Hence

$$
\mathfrak{I} A \subseteq N \cap \not G=1
$$

by hypothesis, proving that

$A$ is torsionfree.

If $g$ is a torsion element in $N$, then $g$ induces a torsion automorphism in $N \cap \mathfrak{H}_{3} G$. Because of (1) we may apply Lemma 4.2 to show that $g \circ\left(N \cap \mathfrak{S}_{3} G\right)=1$. Hence

$$
g \in N \cap \mathfrak{c}_{G}\left(N \cap \mathfrak{H}_{3} G\right)=A ;
$$

and we deduce $g=1$ from (3). Thus we have shown that

$N$ is torsionfree.

For future application we state this as an intermediate result:

(I) If $N \triangleleft G$ with finite $N /\left(N \cap \mathfrak{H}_{3} G\right)$ and $N \cap \mathfrak{P G}=1$, then $N$ is torsionfree.

Denote by $\subseteq$ the set of all $X \triangleleft G$ with $X \subseteq N \cap \mathfrak{S}_{3} G$ and torsionfree $N / X$. It is a consequence of (4) that $1 \in \mathfrak{S}$. Consider a non vacuous subset $\mathfrak{J}$ of $\mathfrak{S}$ with the tower property:

if $X$ and $Y$ belong to $\mathfrak{J}$, then either $X \subseteq Y$ or $Y \subset X$.

The join $T=\bigcup_{X-\mathfrak{I}} X$ is then a normal subgroup of $G$ with $T \subseteq N \cap \mathfrak{H}_{3} G$. If $g \in N$ and $g^{n} \in T$ for some positive integer $n$, then there exists $Y \in \mathfrak{I}$ with $g^{n} \in Y$. But $Y \in \mathfrak{I}$ implies $Y \in \mathfrak{S}$ so that $g \in Y \subseteq T$. Hence $N / T$ is torsionfree so that $T \in \Im$. We have shown that the Maximum Principle of Set Theory may be applied on $\mathfrak{S}$. Consequently there exists a maximal element in $\Xi$, say $M$. We let $G^{*}=G / M$ and $N^{*}=N / M$. Since $M \subseteq N \cap \mathfrak{S}_{3} G$, we have $\mathfrak{H}_{3} G^{*}=\left(\mathfrak{S}_{3} G\right) / M$; and this implies in particular the finiteness of

$$
N^{*} /\left(N^{*} \cap \mathfrak{S}_{3} G^{*}\right)=[N / M] /\left[\left(N \cap \mathfrak{S}_{3} G\right) / M\right] \cong N /\left(N \cap \mathfrak{S}_{3} G\right) .
$$

From $M \in \Xi$ we deduce finally that $N^{*}=N / M$ is torsionfree.

Assume by way of contradiction that $N^{*} \ddagger \mathfrak{G}_{3} G^{*}$. Then $N^{*} /\left(N \cap \mathfrak{S}_{3} G^{*}\right)$ is a finite group, not 1; and since $N^{*}$ is torsionfree, it follows that $N^{*} \cap \mathfrak{S}_{3} G^{*} \neq 1$. Application of the basic properties of the hypercenter shows that

$$
N^{*} \cap_{3} G^{*} \neq 1 \text {. }
$$


Consider $W \triangleleft G^{*}$ with $N^{*} \cap{ }_{3} G^{*} \subseteq W \subseteq N^{*}$ and finite $W /\left(N^{*} \cap{ }_{3} G^{*}\right)$. Since $N^{*}$ is torsionfree, we may apply Lemma 4.1 to show that $W \subseteq 3 G^{*}$. Hence

$$
N^{*} \cap \jmath^{*} \subseteq W \subseteq N^{*} \cap 3 G^{*},
$$

proving that $W /\left(N^{*} \cap{ }_{3} G^{*}\right)=1$. Consequently

$$
1=\left[N^{*} /\left(N^{*} \cap 3 G^{*}\right)\right] \cap \mathfrak{B}\left[G^{*} /\left(N^{*} \cap{ }_{3} G^{*}\right)\right] .
$$

We let $G^{* *}=G^{*} /\left(N^{*} \cap \bigcap_{\jmath} G^{*}\right)$ and $N^{* *}=N^{*} /\left(N^{*} \cap \cap_{\jmath} G^{*}\right)$. Then (6) amounts to

$$
1=N^{* *} \cap \mathbb{P} G^{* *} \text {. }
$$

Since the center is part of the hypercenter, it follows that

$$
\mathfrak{S}_{3} G^{* *}=\mathfrak{S}_{3} G^{*} /\left(N^{*} \cap{ }_{3} G^{*}\right) ;
$$

and this implies

$$
N^{* *} \cap \mathfrak{S}_{3} G^{* *}=\left(N^{*} \cap \mathfrak{S}_{3} G^{*}\right) /\left(N^{*} \cap{ }_{3} G^{*}\right) .
$$

Consequently

(7.b) $N^{* *} /\left(N^{* *} \cap \mathfrak{S}_{3} G^{* *}\right) \cong N^{*} /\left(N^{*} \cap \mathfrak{G}_{3} G^{*}\right) \cong N /\left(N \cap \mathfrak{S}_{3} G\right)$ is finite.

Because of $(7 . \mathrm{a}+\mathrm{b})$ we may apply the intermediate result (I) onto $N^{* *}$ to show that

$$
N^{*} /\left(N^{*} \cap \zeta^{*}\right)=N^{* *} \text { is torsionfree. }
$$

But from (5) and the maximality of $M$ we deduce that $N^{*} /\left(N^{*} \cap{ }_{3} G^{*}\right)$ is not torsionfree, a contradiction proving that

$$
N^{*} \subseteq \mathfrak{H}_{3} G^{*} \text {. }
$$

Hence

so that

$$
1=N^{*} /\left(N^{*} \cap \mathfrak{G}_{3} G^{*}\right) \cong N /\left(N \cap \mathfrak{S}_{3} G\right)
$$

$$
N \subseteq \mathfrak{S}_{3} G .
$$

It is a consequence of (2), (4) and (9) that Lemma 4.3 is true.

Proof of Proposition 4.0. It is quite obvious that (ii) is a consequence of (i). Assume conversely the validity of condition (ii). Consider $K \triangleleft G$ with $N \cap \mathfrak{S}_{3} G \subseteq K \subseteq N$ and finite $K /\left(N \cap \mathfrak{S}_{3} G\right)$. Then $K \cap \mathfrak{S}_{3} G=N \cap \mathfrak{Y}_{3} G$ so that $K /\left(K \cap \mathfrak{S}_{3} G\right)$ is finite; and clearly $K \cap \mathfrak{B} G \subseteq N \cap \mathfrak{B G}=1$. Thus we may apply Lemma 4.3 to show that $K \subseteq \mathfrak{Y}_{3} G$. But $N /\left(N \cap \mathfrak{S}_{3} G\right)$ is, by condition (ii), the product of finite normal subgroups of $G\left(N \cap \mathfrak{H}_{3} G\right)$, contained in $N /\left(N \cap \mathfrak{S}_{3} G\right)$; and their triviality has just been shown. Hence $N /\left(N \cap \mathfrak{S}_{3} G\right)=1$ so that $N \subseteq \mathfrak{H}_{3} G$. Because of $N \cap \mathfrak{B}_{G}=1$ and $N /\left(N \cap \mathfrak{H}_{3} G\right)=1$ we may 
apply Lemma 4.3 a second time to show that $N$ is torsionfree: we have derived (i) from (ii) and shown the equivalence of conditions (i) and (ii).

CO-HYPERCENTRALLY IMBEDDED is the subgroup $S$ of $G$, if $S \triangleleft G$ and if there exists to every $X \triangleleft G$ with $1 \subset X \subseteq S$ a $Y \triangleleft G$ with $Y \subset X$ and $X / Y \subseteq \mathfrak{h} 3(G / Y)$.

This concept will prove too restrictive for most of our needs. To formulate a less restrictive concept we need the following definition.

A PRINCIPAL SUBGROUP of $G$ is every subgroup of the form $\left\{g^{G}\right\}$. These are the normal subgroups, spanned by one element; and our notation should remind the reader of principal ideals.

WEAKLY CO-HYPERCENTRALLY IMBEDDED is the subgroup $S$ of $G$, if $S \triangleleft G$ and $\{X \circ G\} \subset X$ for every principal subgroup $X$ of $G$ with $1 \subset X \subseteq S$. For convenience' sake we shall write $S \mathfrak{w} G$ whenever $S$ is weakly co-hypercentrally imbedded in $G$. To justify this notation and in view of some applications we prove the

LEMMA 4.4. Co-hypercentrally imbedded subgroups are weakly cohypercentrally imbedded.

Proof. Assume that $S$ is a co-hypercentrally imbedded subgroup of $G$. Then $S \triangleleft G$. Consider a principal subgroup $T$ of $G$ with $1 \subset T \subseteq S$. Then there exists a subgroup $U \triangleleft G$ with $U \subset T$ and $1 \subset T / U \subseteq \mathfrak{H}_{3}(G / U)$. Since $T$ is a principal subgroup of $G$, and since $U \subset T$, there exist normal subgroups $X \triangleleft G$ with $U \subseteq X \subset T$; and among these $X$ there exists a maximal one, say $V$ [Maximum Principle of Set Theory]. Because of $U \subseteq V \subset T$ and $T / U \subseteq \mathfrak{H}_{3}(G / U)$ we have $T / V \subseteq \mathfrak{G}_{3}(G / V)$. Because of the maximality of $V$ we find that $T / V$ is a minimal normal subgroup of $G / V$. Hence $T / V \subseteq \mathfrak{H}_{3}(G / V)$ implies $T / V \subseteq \mathfrak{z}(G / V)$; and this is equivalent with

so that

$$
T \circ G \subseteq V
$$

Hence $S \mathfrak{w} G$.

$$
\{T \circ G\} \subseteq V \subset T
$$

LEMMA 4.5. $N \mathfrak{w} G$ and $H \triangleleft G, H \subseteq N \cap \mathfrak{S}_{3} G$ imply $N / H \mathfrak{w} G / H$.

Proof. Denote by $\mathfrak{B}$ the set of all $X \triangleleft G$ with $X \subseteq H$ and $N / X \mathfrak{w} G / X$ From our hypothesis $N$ w $G$ we deduce

$$
1 \in \mathfrak{W} \text {. }
$$

Suppose next that $\mathfrak{B}$ is a non vacuous subset of $\mathfrak{B}$ with the tower property:

if $X$ and $Y$ belong to $\mathfrak{B}$, then either $X \subseteq Y$ or $Y \subset X$. 
The join $J=\bigcup_{X \in \ell} X$ is clearly a normal subgroup of $G$ with $J \subseteq H$. Consider a principal subgroup $A$ of $G / J$ with $A \subseteq N / J$ and $\{A \circ(G / J)\}=A$. There exis.s a principal subgroup $B=\left\{b^{G}\right\}$ of $G$ with $A=B J / J$. C.early $B \subseteq N$ and

$$
B J / J=A=\{A \circ(G / J)\}=\{(B J / J) \circ(G / J)\}=J\{B \circ G\} / J .
$$

Hence $B \subseteq\{B \circ G\} J$ and there exist elements $c \in\{B \circ G\}$ and $j \in J$ with $b=c j$. From the definition of $J$ we deduce the existence of $Y \in \mathfrak{B}$ with $j \in Y$. Hence $b=c j \in\{B \circ G\} Y$ so that

$$
B=\left\{b^{G}\right\} \subseteq\{B \circ G\} Y .
$$

Consequently $B Y / Y$ is a principal subgroup of $G / Y$ with $B Y / Y \subseteq N / Y$ and

$$
B Y / Y=\{B \circ G\} Y / Y=\{(B Y / Y) \circ(G / Y)\} .
$$

Since $Y \in \mathfrak{B}$ belongs to $\mathfrak{B}$, it follows that $N / Y \mathfrak{w} G / Y$; and this implies $B Y / Y=1$. Hence $B \subseteq Y \subseteq J$ so that $A=B J / J=1$; and we have shown that $N / J \mathfrak{w} G / J$. Consequently $J \in \mathfrak{B}$. Thus we have shown [by (1)] that the Maximum Principle of Set Theory may be applied on $\mathfrak{B}$. Hence

there exists a maximal subgroup $M$ in $\mathfrak{M}$.

Assume by way of contradiction that $M \neq H$. From our definition of $\mathfrak{M}$ and our choice of $H$ it follows that

$$
M \subset H \subseteq N \cap \mathfrak{D}_{3} G .
$$

This implies that $1 \subset H / M \subseteq \mathfrak{H}_{3}(G / M)$; and we deduce

$$
1 \neq(H / M) \cap 3(G / M)
$$

from the basic properties of the hypercenter. Denote by $Z$ the uniquely determined subgroup of $G$ with $M \subseteq Z$ and $Z / M=(H / M) \cap 3(G / M)$. Because of (4.a) it follows that

$$
Z \circ G \subseteq M \subset Z \subseteq H \subseteq N \cap \mathfrak{H}_{3} G \text { and } Z \triangleleft G .
$$

Since $M$ is maximal in $\mathfrak{W}$, it follows that $N / Z \mathfrak{w} G / Z$. Hence there exists an element $s$ with the following properties:

$$
s \in N, s \notin Z, Z\left\{s^{G}\right\}=Z\left\{\left\{s^{G}\right\} \circ G\right\} .
$$

We let $S=\left\{s^{G}\right\}$, a principal subgroup of $G$. Because of (5) there exist elements $z \in Z$ and $t \in\{S \circ G\}$ with $s=z t$.

It is a consequence of (4.b) that $z \circ G \subseteq Z \circ G \subseteq M$. Hence

$$
s \circ G=z t \circ G \subseteq(z \circ G)(t \circ G) \subseteq M(t \circ G) \text {. }
$$

Let $D=\left\{t^{G}\right\}$. Then it follows from (6) that 


$$
M D=M\left\{t^{G}\right\} \subseteq M\{S \circ G\}=M\left\{\left\{s^{G}\right\} \circ G\right\} \subseteq M\left\{\left\{t^{G}\right\} \circ G\right\} \subseteq M\{D \circ G\} \subseteq M D .
$$

Hence $M D / M$ is a principal subgroup of $G / M$ with $M D / M \subseteq N / M$ and

$$
M D / M=M\{D \circ G\} / M=\{(M D / M) \circ(G / M)\} .
$$

But $M \in \mathfrak{B}$ by (2) so that $N / M \mathfrak{w} G / M$. Consequently $1=M / M D$ so that $t \in D \subseteq M$. Hence

$$
s=z t \in Z M=Z,
$$

contradicting (5). This contradiction shows that

$$
H=M \in \mathfrak{W},
$$

proving $N / H \mathfrak{w} G / H$.

For a convenient statement of our principal application of this lemma we need the following concept.

NORMALLY DESCENDING SEQUENCES OF SUBGROUPS of $G$ are sequences $N_{i}$ of subgroups of $G$ with

$$
N_{i+1} \subseteq\left\{N_{i} \circ G\right\} \subseteq N_{2} \text { for every } i=1,2, \cdots .
$$

It follows in particular that such a sequence is a descending sequence of normal subgroups of $G$, though this requirement is, in general, somewhat weaker than that of normal descent.

COROLlary 4.6. If $N \mathfrak{w} G$, and if $S$ is a principal subgroup of $G /\left(N \cap \mathfrak{S}_{3} G\right)$ w.th $1 \subset S \subseteq N /\left(N \cap \mathfrak{H}_{3} G\right)$, then
(A) $1 \subset\left\{S \circ\left[G /\left(N \cap \mathfrak{W}_{3} G\right)\right]\right\} \subset S$ and
(B) there exists a normally descending sequence of principal subgroups $S_{i}$ of $G /\left(N \cap \mathfrak{H}_{3} G\right)$ with $S=S_{1}$ and $S_{i+1} \subset S_{1}$ for $0<i$.

Proof. From $N \mathfrak{w} G$ and Lemma 4.5 we deduce that

$$
N /\left(N \cap \mathfrak{H}_{3} G\right) \mathfrak{\mathfrak { O }} G /\left(N \cap \mathfrak{W}_{3} G\right) ;
$$

and this implies in particular that

$$
\left\{S \circ\left[G /\left(N \cap \mathfrak{S}_{3} G\right)\right]\right\} \subset S .
$$

If $\left\{S \circ\left[G /\left(N \cap \mathfrak{S}_{3} G\right)\right]\right\}=1$, then

$$
1 \subset S \subseteq\left[N /\left(N \cap \mathfrak{W}_{3} G\right)\right] \cap_{3}\left[G /\left(N \cap \mathfrak{H}_{3} G\right)\right]=1,
$$

a contradiction proving that

$$
1 \subset\left\{S \circ\left[G /\left(N \cap \mathfrak{S}_{3} G\right)\right]\right\} .
$$

Our contention $(A)$ is an immediate consequence of $(2)+(3)$. 
To prove (B) let $S=S_{1}$ and make the inductive hypothesis that $0<n$ and that $S_{1}, \cdots, S_{n-1}$ are principal subgroups of $G /\left(N \cap \mathfrak{S}_{3} G\right)$ with

$$
1 \subset S_{i+1} \subset S_{i} \text { and } S_{i+1} \subseteq\left\{S_{i} \circ\left[G /\left(N \cap \mathfrak{H}_{3} G\right)\right]\right\} \subseteq S_{i}
$$

for $0<i<n-1$. Then $S_{n-1}$ is a principal subgroup of $G /\left(N \cap \mathfrak{H}_{3} G\right)$ with $1 \subset S_{n-1} \subseteq N /\left(N \cap \mathfrak{H}_{3} G\right)$. Application of (A) shows that

$$
1 \subset\left\{S_{n-1} \circ\left[G /\left(N \cap \mathfrak{H}_{3} G\right)\right]\right\} \subset S_{n-1} .
$$

Consequently there exists an element

$$
1 \neq s \in\left\{S_{n-1} \circ\left[G /\left(N \cap \mathfrak{S}_{3} G\right)\right]\right\}
$$

and it follows from (4) that $S_{n}=\left\{s^{G /(N \cap \mathfrak{s b G})}\right\}$ is a principal subgroup of $G /\left(N \cap \mathfrak{S}_{3} G\right)$ with

$$
1 \subset S_{n} \subseteq\left\{S_{n-1} \circ\left[G /\left(N \cap \mathfrak{S}_{3} G\right)\right]\right\} \subset S_{n-1},
$$

completing the inductive construction of the normally descending sequence of principal subgroups $S_{i}$ of $G /\left(N \cap \mathfrak{S}_{3} G\right)$ with $S=S_{1}$ and $S_{i+1} \subset S_{i}$ for $0<i$. This proves (B).

NOTATIONAL REMINDER. $t X=$ product of all normal torsion subgroups of the group $X$. This is a characteristic torsion subgroup of $X$ with $t X \subseteq \mathfrak{I} X$, though equality will in general not be the case.

THEOREM 4.7. The following properties of $N \triangleleft G$ are equivalent:

(i) $N \subseteq \mathfrak{H}_{3} G$.

(ii) If $N_{i}$ is a normally descending sequence of principal subgroups of $G$ with $N_{i} \subseteq N$, then $N_{i}=1$ for almost all $i$.

(iii) $\left\{\begin{array}{l}\text { (a) } N \mathfrak{w} G \text {. } \\ \text { (b) If } N_{i} \text { is a normally descending sequence of principal subgroups } \\ \text { of } G /\left(N \cap \mathfrak{H}_{3} G\right) \text { with } N_{i} \subseteq N /\left(N \cap \mathfrak{S}_{3} G\right) \text {, then } N_{k}=N_{k+1} \text { for } \\ \text { some } k \text {. }\end{array}\right.$

(iv) $\left\{\right.$ (a) $N /\left(N \cap \mathfrak{H}_{3} G\right) \subseteq \mathfrak{P}\left[G /\left(N \cap \mathfrak{S}_{3} G\right)\right]$.

(b) $\mathfrak{c}_{t N}\left(N \cap \mathfrak{S}_{3} G\right) \subseteq \mathfrak{S}_{3} G$.

(v) $\left\{\right.$ (a) $N /\left(N \cap \mathfrak{H}_{3} G\right) \subseteq \mathfrak{P}\left[G /\left(N \cap \mathfrak{S}_{3} G\right)\right]$.

(b) $\mathfrak{c}_{\mathrm{t} N}\left(N \cap \mathfrak{S}_{3} G\right) \mathfrak{w} G$.

(a) $N /\left(N \cap \mathfrak{H}_{3} G\right) \subseteq \mathfrak{P}\left[G /\left(N \cap \mathfrak{S}_{3} G\right)\right]$.

(b) $\mathfrak{c}_{N \cap \mathfrak{B} G}\left(N \cap \mathfrak{S}_{3} G\right) \subseteq \mathfrak{H}_{3} G$.

(vi) $\{$ (c) If $S$ is a principal subgroup of $G$ with $S=\{S \circ G\} \subseteq \mathfrak{c}_{\mathfrak{t} N}\left(N \cap \mathfrak{H}_{3} G\right)$, then every primary abelian epimorphic image of $S$ is finite. 
Proof. Assume first that $N \subseteq \mathfrak{H}_{3} G$; and consider a normally descending sequence of principal subgroups $S_{i}$ of $G$ with $1 \subset S_{i} \subseteq N$. Denote by $\mathfrak{B}$ the set of all $X \triangleleft G$ with $S_{1} \ddagger X$ for every $i$. From $1 \subset S_{i}$ for every $i$ we deduce that $1 \in \mathfrak{W}$; and from the principality of all the $S_{i}$ we deduce therefore the applicability of the Maximum Principle of Set Theory to $\mathfrak{M}$. Consequently there exists a maximal normal subgroup $M$ in $\mathfrak{M}$. From $S_{i} \neq M$ and $S_{i} \subseteq N$ for every $i$ we deduce that $N \nsubseteq M$. Hence

$$
M \subset M N \subseteq M \mathfrak{H}_{3} G
$$

so that

$$
1 \subset M N / M \subseteq \mathfrak{H}_{3}(G / M) .
$$

Application of the basic properties of the hypercenter shows

$$
1 \subset[M N / M] \cap 3[G / M] \text {. }
$$

If $Z$ is the uniquely determined subgroup of $G$ with $M \subseteq Z$ and $Z / M=[M N / M] \cap_{3}[G / M]$, then

$$
Z \circ G \subseteq M \subset Z \subseteq M N \text { and } Z \triangleleft G \text {. }
$$

Hence $Z \notin \mathfrak{M}$, since $M$ is maximal in $\mathfrak{M}$; and this implies the existence of a positive integer $k$ with $S_{k} \subseteq Z$. Consequently

$$
S_{k+1} \subseteq\left\{S_{k} \circ G\right\} \subseteq\{Z \circ G\} \subseteq M \in \mathfrak{W},
$$

contradicting our definition of $\mathfrak{M}$. This contradiction shows that every normally descending sequence of principal subgroups $S_{i}$ of $G$ with $S_{i} \subseteq N$ contains 1 . But $S_{j}=1$ implies $S_{j+i}=1$ for every positive integer $i$, proving that (ii) is a consequence of (i).

Assume next the validity of (ii). If $K$ is a principal subgroup of $G$ with $K=\{K \circ G\} \subseteq N$, then the sequence $S_{i}=K$ for every $i$ is a normally descending sequence of principal subgroups of $G$ with $S_{i} \subseteq N$. Hence $K=S_{i}=1$ for some $i$ [by (ii)]; and this proves $N$ w G.--Next we deduce from (ii) that every normally descending sequence of principal subgroups $S_{i}$ of $G$ with $S \subseteq N$ terminates after finitely many steps; and this implies the corresponding statement for $N /\left(N \cap \mathfrak{H}_{3} G\right) \triangleleft G /\left(N \cap \mathfrak{H}_{3} G\right)$. It follows that not only (iii.a), but also (iii.b) is a consequence of (ii): we have derived (iii) from (ii).

If (iii) is satisfied by $N$, and if $N \nsubseteq \mathfrak{H}_{3} G$, then $N \cap \mathfrak{H}_{3} G \subset N$ so that $1 \neq N /\left(N \cap \mathfrak{S}_{3} G\right)$. This implies the existence of a principal subgroup $S$ of $G /\left(N \cap \mathfrak{S}_{3} G\right)$ with $1 \subset S \subseteq N /\left(N \cap \mathfrak{H}_{3} G\right)$. Because of (iii.a) we may apply Corollary 4.6,(B) to obtain a contradiction to our condition (iii.b). This contradiction shows that $N \subseteq \mathfrak{H}_{3} G$. Thus (i) is a consequence of (iii), completing the proof of the equivalence of conditions (i)-(iii). 
If (i) is satisfied by $G$, then $N /\left(N \cap \mathfrak{S}_{3} G\right)=1$, implying the validity of (iv.a); and (iv.b) is true, since every subgroup of $N \subseteq \mathfrak{H}_{3} G$ is part of the hypercenter of $G$. Hence (iv) is a consequence of (i).

Assume conversely the validity of (iv). Let $G^{*}=G / \mathrm{t} N$ and $N^{*}=N / \mathrm{t} N$. Then $N^{*} \triangleleft G^{*}$ and we deduce

$$
N^{*} /\left(N^{*} \cap \mathfrak{H}_{3} G^{*}\right) \subseteq \mathfrak{P}\left[G^{*} /\left(N^{*} \cap \mathfrak{H}_{3} G^{*}\right)\right]
$$

from (iv.a) and $\mathfrak{S}_{3} G \mathrm{t} N / \mathrm{t} N \subseteq \mathfrak{S}_{3} G^{*}$. Next we note that $N^{*} \cap \mathfrak{P} G^{*}$ is a normal torsion subgroup of $G^{*}$. Since extensions of torsion groups by torsion groups are torsion groups, we conclude that

$$
N^{*} \cap \mathfrak{B} G^{*}=1 .
$$

Thus Proposition 4.0 may be applied upon $N^{*} \triangleleft G^{*}$ so that $N^{*}$ [is torsionfree and]

$$
N / \mathrm{t} N=N^{*} \subseteq \mathfrak{H}_{3} G^{*}=\mathfrak{H}_{3}(G ; \mathrm{t} N) .
$$

Denote by $\Gamma$ the group of automorphism, induced by $G$ in $N \cap \mathfrak{S}_{3} G$. Then $\Gamma \cong G / \mathfrak{c}_{\mathfrak{G}}\left(N \cap \mathfrak{H}_{3} G\right)$; and $\Gamma$ stabilizes the factors

$$
\left(N \cap{z_{\sigma}+1}_{1} G\right) /\left(N \cap{ }_{z_{\sigma}} G\right)
$$

where the $\beta_{\sigma} G$ [with $\sigma$ ranging over finite and transfinite ordinals] are the terms of the ascending central chain of $G$. Hence we may apply Hall-Hartley [p. 5, Theorem A.1] to show that $\Gamma$ and hence

$$
G / \mathcal{c}_{G}\left(N \cap \mathfrak{H}_{3} G\right) \text { possesses a descending hypercentral series }
$$

as defined by Hall and Hartley [pp. 1-2]. It is a consequence of (4) that the normal subgroup $N \mathfrak{c}_{G}\left(N \cap \mathfrak{H}_{3} G\right) / \mathfrak{c}_{G}\left(N \cap \mathfrak{H}_{3} G\right)$ is a co-hypercentrally imbedded subgroup of $G / \mathfrak{c}_{G}\left(N \cap \mathfrak{S}_{3} G\right)$; and this implies by Lemma 4.4 that

$$
N \mathfrak{c}_{\mathfrak{G}}\left(N \cap \mathfrak{S}_{3} G\right) / \mathfrak{c}_{G}\left(N \cap \mathfrak{S}_{3} G\right) \mathfrak{w} G / \mathfrak{c}_{G}\left(N \cap \mathfrak{S}_{3} G\right) .
$$

Since the canonical isomorphism

$$
N \mathfrak{c}_{\mathfrak{G}}\left(N \cap \mathfrak{H}_{3} G\right) / \mathfrak{c}_{G}\left(N \cap \mathfrak{H}_{3} G\right) \cong N /\left[N \cap \mathfrak{c}_{G}\left(N \cap \mathfrak{H}_{3} G\right)\right]=N / \mathfrak{c}_{N}\left(N \cap \mathfrak{H}_{3} G\right)
$$

is a $G$-isomorphism, we conclude that

$$
N / \mathfrak{c}_{N}\left(N \cap \mathfrak{G}_{3} G\right) \mathfrak{w} G / \mathfrak{c}_{N}\left(N \cap \mathfrak{W}_{3} G\right) .
$$

Let $\tilde{G}=G / \mathfrak{c}_{N}\left(N \cap \mathfrak{S}_{3} G\right)$ and $\tilde{N}=N / \mathfrak{c}_{N}\left(N \cap \mathfrak{H}_{3} G\right)$. Then we deduce

$$
\tilde{N} \text { w } \tilde{G}
$$

from (5). From $\mathfrak{S}_{3} G_{\mathfrak{c}_{N}}\left(N \cap \mathfrak{S}_{3} G\right) / \mathfrak{c}_{N}\left(N \cap \mathfrak{H}_{3} G\right) \subseteq \mathfrak{S}_{3} \widetilde{G}$ and condition (iv.a) we deduce that 


$$
\tilde{N} /\left(\tilde{N} \cap \mathfrak{H}_{3} \tilde{G}\right) \subseteq \mathfrak{B}\left[\tilde{G} /\left(\tilde{N} \cap \mathfrak{S}_{3} \tilde{G}\right)\right] .
$$

Thus every principal subgroup of $\tilde{G} /\left(\tilde{N} \cap \mathfrak{H}_{3} \tilde{G}\right)$ which is part of $\tilde{N} /\left(\tilde{N} \cap \mathfrak{H}_{3} \tilde{G}\right)$ is finite. Combine this remark with $(\tilde{5})$ to show that our condition (iii) is satisfied by $\tilde{N} \triangleleft \tilde{G}$. Since we have already verified the equivalence of (i) and (iii), it follows that

$$
N / \mathfrak{c}_{N}\left(N \cap \mathfrak{H}_{3} G\right)=\tilde{N} \subseteq \mathfrak{S}_{3} \tilde{G}=\mathfrak{h}_{3}\left[G / \mathfrak{c}_{N}\left(N \cap \mathfrak{H}_{3} G\right)\right]
$$

Combination of (3), (7) and Lemma 1.6 shows the valid ty of

$$
\begin{aligned}
N / \mathfrak{c}_{\mathrm{t} N}\left(N \cap \mathfrak{H}_{3} G\right) & =N /\left[\operatorname{t} N \cap \mathfrak{c}_{N}\left(N \cap \mathfrak{S}_{3} G\right)\right] \\
& \subseteq \mathfrak{H}_{3}\left[G /\left(\mathrm{t} N \cap \mathfrak{c}_{N}\left[N \cap \mathfrak{S}_{3} G\right]\right)\right] \\
& \subseteq \mathfrak{H}_{3}\left[G / \mathfrak{c}_{\mathrm{t} N}\left(N \cap \mathfrak{S}_{3} G\right)\right] .
\end{aligned}
$$

Now we apply condition (iv.b) for the first time. Recalling that $\mathfrak{H}_{3}\left[X / \mathfrak{G}_{3} X\right]=1$ we deduce from (8) and (iv.b) that $N \subseteq \mathfrak{H}_{3} G$ : we have derived (i) from (iv), proving the equivalence of (i)-(iv).

It is clear that ( $v$ ) is a consequence of (iv) and Lemma 4.4. If conversely (v) is satisfied by $N \triangleleft G$, then we deduce from (v.a) and Lemma 1.7 the validity of

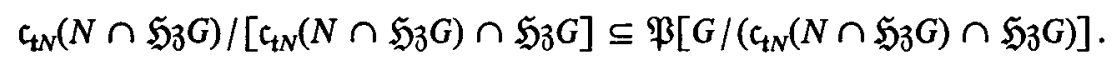

It follows that every principal subgroup of $G /\left[\mathfrak{c}_{t_{N}}\left(N \cap \mathfrak{S}_{3} G\right) \cap \mathfrak{H}_{3} G\right]$ which is part of $\mathfrak{c}_{\mathfrak{t} N}\left(N \cap \mathfrak{S}_{3} G\right) /\left[\mathfrak{c}_{\mathfrak{T}}\left(N \cap \mathfrak{S}_{3} G\right) \cap \mathfrak{S}_{3} G\right]$ is finite. Thus condition (iii.b) is satisfied by $\mathfrak{C}_{W N}\left(N \cap \mathfrak{S}_{3} G\right) \triangleleft G$; and that condition (iii.a) is satisfied, is the content of our present condition (v.b). Hence we may apply the equivalence of conditions (i) and (iii) to show that $c_{\mathrm{t} N}\left(N \cap \mathfrak{S}_{3} G\right) \subseteq \mathfrak{H}_{3} G$ : we have derived condition (iv) from (v), proving the equivalence of conditions (i)-(v).

It is quite obvious that (vi) is a consequence of the equivalent properties (i)-(v). We assume conversely the validity of condition (vi) and consider a principal subgroup $S$ of $G$ with $S=\{S \circ G\} \subseteq \mathfrak{c}_{\mathfrak{t}_{N}}\left(N \cap \mathfrak{S}_{3} G\right)$. Then

so that

$$
S \circ\left(S \cap \mathfrak{H}_{3} G\right) \subseteq \mathfrak{C}_{\mathfrak{t}}\left(N \cap \mathfrak{H}_{3} G\right) \circ\left(\mathfrak{t} N \cap \mathfrak{H}_{3} G\right)=1
$$

$$
S \cap \mathfrak{S}_{3} G \subseteq{ }_{3} S .
$$

Next we note that

$$
S /\left(S \cap \mathfrak{S}_{3} G\right) \cong S_{\mathfrak{y}_{3}} G / \mathfrak{S}_{3} G \subseteq N \mathfrak{S}_{3} G / \mathfrak{S}_{3} G \cong N /\left(N \cap \mathfrak{S}_{3} G\right)
$$

and it follows from condition (vi.a) and the fact that these isomorphisms are $G$-isomorphisms that $S /\left(S \cap \mathfrak{H}_{3} G\right)$ is a product of finite normal subgroups of $G /\left(S \cap \mathfrak{S}_{3} G\right)$. From the principality of $S$ we deduce therefore that

$$
S /\left(S \cap \mathfrak{S}_{3} G\right) \text { is finite. }
$$


Combine (10.a) and (10.b) to see that $S / \not S$ is finite. Thus we may apply a Theorem of I. Schur to show that

$$
S^{\prime} \text { is finite; }
$$

see Huppert [p. 417, 2.3 Satz].

Since $S$ is a principal torsion group, the abelian group $S / S^{\prime}$ is generated by a set of torsion elements of equal order. It follows that $S / S^{\prime}$ is a direct product of cyclic groups of bounded order; see Specht [p. 279, Satz 4]. Application of condition (vi.c) shows that every primary elementary abelian epimorphic image of $S / S^{\prime}$ is finite. Consequently

$S / S^{\prime}$ is finite.

Combine (10.c) and (10.d) to see that

$S$ is finite.

Hence it follows from condition (vi.b) that

$$
S \subseteq \mathfrak{c}_{I_{N}}\left(N \cap \mathfrak{H}_{3} G\right) \cap \mathfrak{P G}=\mathfrak{c}_{N \cap \mathfrak{B G}}\left(N \cap \mathfrak{H}_{3} G\right) \subseteq \mathfrak{S}_{3} G .
$$

Thus it follows from Lemma 4.4 that $S \mathfrak{w} G$. Since $S=\{S \circ G\}$, it follows that $S=1$; and thus we have shown that

$$
\mathfrak{c}_{\mathfrak{t}}\left(N \cap \mathfrak{H}_{3} G\right) \mathfrak{w} G .
$$

Since (10) $=$ (iv.b) and (vi.a) = (iv.a), we have deduced condition (iv) from (vi), proving the equivalence of conditions (i)-(vi).

Discussion 4.8. A. It is readily seen that every non-abelian free group $F$ satisfies $\mathfrak{h}_{3} F=1$; and it is a consequence of a Theorem of Magnus - see Specht [p. 211, Satz 21] - that $F \mathfrak{w} F$. This shows the indispensability of conditions (iii.b) and (v.a) of Theorem 4.7.

B. Every finite group and more generally every group with minimum condition for normal subgroups satisfies condition (iii.b) of Theorem 4.7, though such groups will, in general, not be hypercentral. This shows the indispensability of condition (iii.a) of Theorem 4.7.

C. Denote by $p$ an odd prime and by $A$ a countably infinite elementary abelian $p$-group. Then $A=B \times\{a\}$ is the direct product of a cyclic subgroup $\{a\}$ of order $p$ and a subgroup $B \cong A$.

Denote by $\Delta$ the set of all automorphisms $\sigma$ of $A$ with

$$
B^{\sigma-1}=1 \text { and } a^{\sigma-1} \in B .
$$

It is readily seen that $\Delta$ is a group isomorphic to $B$ and hence a countably infinite, elementary abelian $p$-group. 
Denote by $\lambda$ the uniquely determined automorphisms of $A$ with

$$
B^{\lambda-1}=1 \text { and } a^{\lambda}=a^{-1} \text {. }
$$

Then $o(\lambda)=2$ and $\lambda \sigma \lambda=\sigma^{-1}$ for every $\sigma \in \Delta$. Thus $\Delta$ is a normal subgroup of $\Gamma=\{\Delta, \lambda\}=\Delta\{\lambda\}$ with $[\Gamma: \Delta]=2$; and $\Gamma$ is a torsion group the orders of whose elements are $1,2, p$.

Finally we form the product $G=A \Gamma$ in the holomorph of $A$. Then one verifies easily that

$$
B={ }_{3} G=\mathfrak{H}_{3} G, A \triangleleft G \text { and } A / B=A /\left(A \cap \mathfrak{H}_{3} G\right) \text { is cyclic of order } p .
$$

Thus condition (iv.a) of Theorem 4.7 is satisfied by $A \triangleleft G$, proving the indispensability of condition (iv.b) of Theorem 4.7.

It is easily verified that $A$ is the only normal subgroup of $G$ which is part of $A$, but not part $B$. Consequently

$$
B=\mathfrak{B} G=\mathfrak{Q} G \subset A .
$$

D. The indispensability of condition (iv.a) $=(v . a)=($ vi.a) is shown by every infinite torsionfree group $G$ with $\mathfrak{H}_{3} G=1$.

E. The indispensability of condition (vi.b) is shown by every normal subgroup of a finite group $G$ which is not part of $\mathfrak{H}_{3} G$.

F. The indispensability of condition (vi.c) may be seen by consideration of the example, constructed ad $\mathbf{C}$.

\section{The $\mathfrak{Q}$-imbedded normal subgroups}

We shall need a slightly weaker relation than the relation $\mathfrak{w}$, discussed in section 4. Accordingly we define $S \mathfrak{w}_{\infty} G$ if, and only if, $S \triangleleft G$ and $\{X \circ G\} \subset X$ whenever $X$ is an infinite principal subgroup of $G$ with $X \subseteq S$.

One may say that $\mathfrak{w}_{\infty}$ is the restriction of $\mathfrak{w}$ to infinite principal subgroups $X$ [instead of principal subgroups, not 1].

LEMMA 5.1.: If $N \triangleleft G$, then $N \mathfrak{w}_{\infty} G$ is necessary and sufficient for

$$
N /(N \cap \mathfrak{P} G) \mathfrak{w} G /(N \cap \mathfrak{P} G) .
$$

Proof. Assume first that $N /(N \cap \mathfrak{B} G) \mathfrak{w} G /(N \cap \mathfrak{B} G)$; and consider an infinite principal normal subgroup $S$ of $G$ with $S \subseteq N$. Every element in $\mathfrak{P G}$ is, by definition, contained in a finite normal subgroup of $G$. Thus a principal subgroup of $G$ is part of $\mathfrak{P} G$ if, and only if, it is finite. Consequently $S \nsubseteq \mathfrak{B} G$. Hence $S(N \cap \mathfrak{P} G) /(N \cap \mathfrak{P G})$ is a principal subgroup, not 1 , of $G /(N \cap \mathfrak{P G})$ which is part of $N /(N \cap \mathfrak{B} G) \mathfrak{w} G /(N \cap \mathfrak{P G})$. This implies 


$$
\begin{aligned}
\{S \circ G\} & (N \cap \mathfrak{P G}) /(N \cap \mathfrak{P} G)= \\
& =\{[S(N \cap \mathfrak{P} G) /(N \cap \mathfrak{P} G)] \circ[G /(N \cap \mathfrak{P} G)]\} \\
& \subset S(N \cap \mathfrak{P G}) /(N \cap \mathfrak{P} G) .
\end{aligned}
$$

Hence $\{S \circ G\} \subset S$, proving $N \mathfrak{w}_{\infty} G$.

Assume conversely the validity of $N \mathfrak{w}_{\infty} G$; and consider the set $\mathfrak{B}$ of all pairs $A, B$ with the following properties:

(0) $A$ is a finite normal subgroup of $G$ and $B$ is an infinite principal subgroup of $G$ with $A\{B \circ G\}=B \subseteq N$.

If $\mathfrak{M}$ were not vacuous, then there would exist among the pairs $A, B$ in $\mathfrak{W}$ one with first coordinate of minimal order, say $E, F$. From our hypothesis $N \mathfrak{w}_{\infty} G$ and from $E, F \in \mathfrak{B}$ we deduce

$$
\{F \circ G\} \subset F \text {. }
$$

Since $F=E\{F \circ G\}$ is principal,

(2) there exist elements $e \in E$ and $d \in\{F \circ G\}$ with $F=\left\{(e d)^{G}\right\}$ and furthermore it follows that

$$
\begin{gathered}
\{F \circ G\}=\{E \circ G\}\{\{F \circ G\} \circ G\} ; \\
F=E\{F \circ G\}=E\{E \circ G\}\{\{F \circ G\} \circ G\}=E\{\{F \circ G\} \circ G\},
\end{gathered}
$$

[since $E \triangleleft G$ ].

Assume by way of contradiction that $E=\{E \circ G\}$. Then we deduce from (3) and (4) that

$$
F=E\{\{F \circ G\} \circ G\}=\{E \circ G\}\{\{F \circ G\} \circ G\}=\{F \circ G\},
$$

contradicting (1). Hence

$$
\{E \circ G\} \subset E .
$$

From (2) we deduce that

Hence

$$
F=\left\{(e d)^{G}\right\} \subseteq\left\{e^{G}\right\}\left\{d^{G}\right\} \subseteq E\left\{d^{G}\right\} \subseteq E\{F \circ G\}=F .
$$

$$
F=\left\{e^{G}\right\}\left\{d^{G}\right\}=E\left\{d^{G}\right\}=E\{F \circ G\} .
$$

From (2) and (6) it follows that

$$
\left\{\left\{d^{G}\right\} \circ G\right\} \subseteq\left\{d^{G}\right\} \subseteq\{F \circ G\}=\{E \circ G\}\left\{\left\{d^{G}\right\} \circ G\right\} .
$$

Application of Dedekind's Modular Law shows therefore

$$
\left\{d^{G}\right\}=\left\{\left\{d^{G}\right\} \circ G\right\}\left[\{E \circ G\} \cap\left\{d^{G}\right\}\right] .
$$

It is a consequence of (5) that

$$
\{E \circ G\} \cap\left\{d^{G}\right\} \subseteq\{E \circ G\} \subset E ;
$$


and thus it follows from the minimality of the pair $E, F$ in $\mathfrak{M}$ that the pair $\{E \circ G\} \cap\left\{d^{G}\right\},\left\{d^{G}\right\}$ does not belong to $\mathfrak{W}$. Since $\left\{d^{G}\right\} \subseteq\{F \circ G\} \subseteq F \subseteq N$ [by (0)], comparison of (0) and (7) shows that $\left\{d^{G}\right\}$ is finite. Since $E$ is finite, we deduce from (6) the finiteness of $F$, a contradiction proving that the set $\mathfrak{B}$ is empty.

Suppose now that $V$ is a principal subgroup of $G /(N \cap \mathfrak{P G})$ with

$$
1 \subset V \subseteq N /(N \cap \mathfrak{P} G) \text { and } V=\{V \circ[G /(N \cap \mathfrak{P} G)]\} \text {. }
$$

There exists a principal subgroup $S$ of $G$ with $V=S(N \cap \mathfrak{P G}) /(N \cap \mathfrak{P G})$. Clearly

$$
S \subseteq N, S \nsubseteq \mathfrak{P G} ;
$$

and this implies in particular that

$S$ is infinite.

From $V=\{V \circ[G /(N \cap \mathfrak{P} G)]\}$ we deduce furthermore

$$
S(N \cap \mathfrak{P G})=\{S \circ G\}(N \cap \mathfrak{B G}) .
$$

Since $S$ is principal, we deduce from (9.c) the existence of elements $s \in\{S \circ G\}$, $t \in N \cap \mathfrak{B} G$ with $S=\left\{(s t)^{G}\right\}$. It follows that

$$
\{S \circ G\} \subseteq S \subseteq\left\{s^{G}\right\}\left\{t^{G}\right\} \subseteq\{S \circ G\}\left\{t^{G}\right\} ;
$$

and application of Dedekind's Modular Law shows therefore that

$$
S=\{S \circ G\}\left[S \cap\left\{t^{G}\right\}\right] \text {. }
$$

But $t \in \mathfrak{P} G$ so that $\left\{t^{G}\right\}$ and a fortiori $S \cap\left\{t^{G}\right\}$ is a finite normal subgroup of $G$. Since $S$ is by $(9 . \mathrm{a}+\mathrm{b})$ an infinite principal subgroup of $G$ which is part of $N$, we deduce from (9.d) and the finiteness of $S \cap\left\{t^{\bullet}\right\}$ that the pair $S \cap\left\{t^{G}\right\}, S$ belongs to $\mathfrak{W}$; see $(0)$. But $\mathfrak{W}$ is, by $(8)$, vacuous. Thus we have arrived at a contradiction proving that $N /(N \cap \mathfrak{B} G) \mathfrak{w} G /(N \cap \mathfrak{B} G)$, as we wanted to show.

THEOREM 5.2. The following properties of $N \triangleleft G$ are equivalent:

(i) $\quad N \subseteq \mathbb{Q}$.

(ii) If $S_{i}$ is a normally descending sequence of principal subgroups of $G$ with $S . \subseteq N$, then almost all $S_{i}$ are finite.

$$
\left\{\begin{array}{l}
\text { (a) } N \mathrm{w}_{\infty} G . \\
\text { (b) If } S_{i} \text { is a normally descending sequence of principal subgroups } \\
\text { of } G \text { with } S_{1} \subseteq N \text {, then } S_{i}=S_{i+1} \text { for some } i .
\end{array}\right.
$$


(a) Every normally descending sequence of principal subgroups $S_{i}$ of $G$ with $S_{1} \subseteq N$ terminates after finitely many steps.

$\{$ (b) If $A$ is a principal subgroup of $G$ with $A=\{A \circ G\} \subseteq N$, and if $A=\left\{x^{G}\right\}$ for every $x \in A$ with $x \notin \mathbb{Q} G$, then there exists an element $a$ with finite $a^{G}$ and $A=\left\{a^{G}\right\}$.

(v)

(a) $N \mathfrak{w}_{\infty} G$.

(b) If $S_{i}$ is a normally descending sequence of principal subgroups of $G /(N \cap \mathfrak{Q} G)$ with $S_{1} \subseteq N /(N \cap \mathfrak{Q} G)$, then $S_{k}=S_{k+1}$ for some $k$.

$\left\{\begin{array}{l}\text { (a) } c_{t N}[(N \cap \mathbb{Q} G) /(t N \cap \mathfrak{P} G)] \subseteq \mathfrak{Q} G . \\ \text { (b) } N /(N \cap \mathbb{Q} G) \subseteq \mathfrak{P}[G /(N \cap \mathbb{Q} G)] \text {. }\end{array}\right.$
(a) $\mathfrak{c}_{\mathfrak{t}}[(N \cap \mathfrak{Q} G) /(\mathrm{t} N \cap \mathfrak{P G})] \mathfrak{w}_{\infty} G$.
(b) $N /(N \cap \mathbb{Q} G) \subseteq \mathfrak{P}[G /(N \cap \mathbb{Q} G)]$.

Proof. From $\mathfrak{Q} G / \mathfrak{P} G=\mathfrak{H} 3(G / \mathfrak{B} G)$ and Corollary 3.3, (a) one deduces readily the equivalence of $N \subseteq \mathbb{Q} G$ with

$$
N /(N \cap \mathfrak{B} G) \subseteq \mathfrak{h}_{3}[G /(N \cap \mathfrak{P} G)] .
$$

Next we note that $N \cap \mathfrak{P G}$ is a normal torsion subgroup of $N$. Hence $N \cap \mathfrak{P G} \subseteq \mathrm{t} N$. Since extensions of torsion groups by torsion groups are torsion groups, we conclude that

$$
N \cap \mathfrak{P G} \subseteq \mathrm{t} N \text { and } \mathrm{t} N /(N \cap \mathfrak{P} G)=\mathrm{t}[N /(N \cap \mathfrak{P} G)]
$$

Assume now the validity of ( $\mathrm{i}$; and consider a normally descending sequence of principal subgroups $S_{i}$ of $G$ with $S_{1} \subseteq N$. Then $T_{i}=S_{i}(N \cap \mathfrak{P} G) /(N \cap \mathfrak{P} G)$ is a normally descending sequence of principal subgroups of $G /(N \cap \mathfrak{P G})$ with

$$
T_{1} \subseteq N /(N \cap \mathfrak{P G}) \subseteq \mathfrak{H}_{3}[G /(N \cap \mathfrak{P G})]
$$

by (i*). Application of Theorem 4.7 shows that $T_{i}=1$ for almost all $i$; and this is equivalent with $S_{i} \subseteq \mathfrak{P G}$ for almost all $i$. Since every element in $\mathfrak{B G}$ belongs to a finite normal subgroup of $G$, and since every $S_{i}$ is principal, it follows that $S_{i}$ is finite for almost all $i$ : we have deduced (ii) from (i).

Assume next the validity of (ii) and consider a prin ipal subgroup $S$ of $G$ with $S=\{S \circ G\} \subseteq N$. Then the sequence $S_{i}=S$ for all $i$ is a normally descending sequence of principal subgroups of $G$ with $S_{1}=S \subseteq N$; and we deduce from (ii) that almost all $S_{i}$ are finite. Hence $S$ is finite; and we have deduced $N \mathfrak{w}_{\infty} G$ from (ii). Furth rmore it is clear that the following property is a consequence of (ii):

(iii.b*) Every normally descending sequence of principal subgroups $S_{i}$ of $G$ with $S_{1} \subseteq N$ terminates after finitely many steps.

Since (iii.b) is just a weak form of (iii.b*), we have deduced (iii) from (ii). 
Assume next the validity of (iii) and consider a normally descending sequence of principal subgroups $T_{i}$ of $G /(N \cap \mathfrak{B} G)$ with $T_{1} \subseteq N /(N \cap \mathfrak{B} G)$. One constructs by complete induction a normally descending sequence of principal subgroups $S_{\imath}$ of $G$ with

$$
T_{i}=S_{i}(N \cap \mathfrak{P G}) /(N \cap \mathfrak{P} G) \text { for every } i .
$$

It is clear that $S_{1} \subseteq N$. Application of condition (iii.b) shows therefore the existence of an integer $k$ with $S_{k}=S_{k+1}$. Hence

$$
S_{k}=S_{k+1} \subseteq\left\{S_{k} \circ G\right\} \subseteq S_{k}
$$

so that $S_{k}=\left\{S_{k} \circ G\right\} \subseteq N$. Application of condition (iii.a) shows that $S_{k}$ is finite. Hence $S_{k} \subseteq N \cap \mathfrak{P G}$ so that $T_{k}=1$ : we have shown that $N /(N \cap \mathfrak{B G})$ satisfies condition (ii) of Theorem 4.7. Henc: $N /(N \cap \mathfrak{B} G) \subseteq \mathfrak{h}_{3}[G /(N \cap \mathfrak{P G})]$ : we have deduced ( $\left.i^{*}\right)$ from (iii). Thus (i) is a consequence of (iii), proving the equivalence of conditions (i)-(iii).

If the equivalent conditions (i)-(iii) are satisfied by $N \triangleleft G$, then condition (iii.b*) $=$ (iv.a) is likewise satisfied. If furthermore $S$ is a principal subgroup of $G$ with $S=\{S \circ G\} \subseteq N$, then we deduce the finiteness of $S$ from (iii.a); and this implies the validity of (iv.b): we have deduced condition (iv) from the equivalent conditions (i)-(iii).

Assume the validity of condition (iv). Denote by $\mathfrak{M}$ the set of all principal subgroups $S$ of $G$ with the property:

$$
S=\{S \circ G\} \subseteq N .
$$

Then we may apply condition (iv.a) onto every descending sequence of subgroups in $\mathfrak{M}$, since such a sequence is a normally descending sequence of principal subgroups of $G$, contained in $N$. Thus all such sequences terminate after finitely many steps. This is equivalent with the following property:

(2) The minimum condition is satisfied by the principal subgroups in $\mathfrak{M}$.

Consider an element $e \in G$ with $e^{G}$ a finite class of conjugate elements and the further property that $\left\{x^{G}\right\} \subset\left\{e^{G}\right\}$ implies $x \in \mathbb{Q} G$. This last cond.tion is equivalent with the property:

$$
X \triangleleft G \text { and } X \subset\left\{e^{G}\right\} \text { imply } X \subseteq \mathbb{Q} G \text {. }
$$

Let $E=\left\{e^{G}\right\}$. Then we deduce from the finiteness of $e^{G}$ and Lemma 3.1, $(\mathrm{A} . \mathrm{b}+\mathrm{c})$ that

(3.b) $\mathfrak{T} E$ is a finite normal subgroup of $G$ and that $\widetilde{E}=E / \mathfrak{T} E$ is free abelian of finite rank.

If firstly $\widetilde{E}=1$, then $E=\mathfrak{I} E \subseteq \mathfrak{B} G \subseteq \mathfrak{Q} G$. 
If secondly $\widetilde{E} \neq 1$, then $\widetilde{E}^{n} \subset \widetilde{E}$ for every integer $n$ with $1<n$. Denote by $E_{n}$ the uniquely determined subgroup of $G$ with $\mathfrak{I} E \subseteq E_{n}$ and $E_{n} / \mathfrak{T} E=\tilde{E}^{n}$. Since $\widetilde{E}^{n}$ is a characteristic subgroup of $\tilde{E} \triangleleft G / \mathfrak{I} E$, it follows that $E_{n} \triangleleft G$ and $E_{n} \subset E$. Thus it follows from (3.a) that $E_{n} \subset \mathbb{Q} G$. Application of (3.b) shows that $\tilde{E}=\prod_{1<n} \widetilde{E}^{n}$. Hence $E=\prod_{1<n} E_{n} \subseteq \mathbb{Q} G$. Thus we have shown the following fact:

(3) If $e \in G$ with finite $e^{G}$, and if $\left\{x^{G}\right\} \subset\left\{e^{G}\right\}$ implies $x \in \mathfrak{Q} G$, then $e \in \mathbb{Q} G$.

Denote by $\mathfrak{M}^{*}$ the set of all $X \in \mathfrak{M}$ with $X \nsubseteq \mathfrak{Q} G$. Consider a principal subgroup $W$ of $G$ with $W \subseteq N$ and $W \ddagger Q Q G$. Then there exists $w$ with $W=\left\{w^{G}\right\}$; and clearly $w \notin \mathbb{Q} G$. Consequently there exists by Theorem 3.2 a $G$-commutator sequence $c_{i}$ with $w=c_{1}$ and $c_{i} \neq c_{j}$ for $i \neq j$. If one of the $c_{i}$ were in $\mathfrak{Q} G$, then a second application of Theorem 3.2 would show that the $G$-commutator sequence $c_{n}$ is finitely valued, an impossibility. Hence

$$
c_{i} \notin \mathbb{Q} G \text { for every } i \text {. }
$$

Since $c_{1}=w \in N \triangleleft G$, and since the $c_{i}$ form a $G$-commutator sequence, it follows th $\mathrm{t}$

$$
c_{i} \in N \text { for every } i .
$$

Let $C_{i}=\left\{c_{i}^{G}\right\}$. Then we deduce from $c_{i+1} \in c_{i} \circ G$ that the $C_{i}$ form a normally descending sequence of principal subgroups of $G$ with $C_{i} \subseteq N$ by (4.b). Application of (iv.a) shows that this sequence terminates after finitely many steps. Hence there exists a positive integer $k$ with

$$
C_{k}=C_{k+1} \subseteq\left\{C_{k} \circ G\right\} \subseteq C_{k} .
$$

Thus $C_{k}=\left\{C_{k} \circ G\right\} \subseteq C_{1}=W$; and $C_{k} \nsubseteq \mathbb{Q} G$ by (4.a). Thus we have shown:

(4) If $W$ is a principal subgroup of $G$ with $W \subseteq N$ and $W \nsubseteq Q Q G$, then there exists $V \in \mathfrak{M}^{*}$ with $V \subseteq W$.

Assume now by way of contradiction that

$$
N \nsubseteq \mathfrak{Q} G \text {. }
$$

Then there exists an element $w \in N$ with $w \notin Q Q G$; and we deduce from (4) the existence of $V \in \mathfrak{M}^{*}$ with $V \subseteq\left\{w^{\cup}\right\}$. This implies in particular that

$$
\mathfrak{M}^{*} \text { is not vacuous. }
$$

Since $\mathfrak{M}^{*}$ is part of $\mathfrak{M}$ [by definition], we deduce from (2) and (6) the existence of a minimal subgroup $L$ in $\mathfrak{M}^{*}$. If $x \in L$, but $x \notin \mathbb{Q} G$, then $x \in N$; and we deduce from (4) the existence of $X \in \mathfrak{M}^{*}$ with $X \subseteq\left\{x^{\cup}\right\}$. Then $X \subseteq L$ and we deduce

$$
L=X \subseteq\left\{x^{G}\right\} \subseteq L
$$

from the minimality of $L$ so that $L=\left\{x^{G}\right\}$ for every $x \in L, x \notin Q G$. Thus we may 
apply condition (iv.b) on $L$. Consequently there exists $e$ with finite $e^{\mathrm{G}}$ and $L=\left\{e^{6}\right\}$. We may apply (3) on $L$ showing that $L \subseteq \mathfrak{Q} G$, contradicting $L \in \mathfrak{M}^{*}$. This contradiction shows the absurdity of our assumption (5); and we have shown that $N \subseteq \mathbb{Q} G$ is a consequence of condition (iv), proving the equivalence of conditions (i)-(iv).

If conditions (i)-(iv) are satisfied by $N$, then we note that (v.a) $=$ (iii.a) and that $N /(N \cap Q G)=1$ [by (i)], showing the validity of (v). If conversely condition (v) is satisfied by $N$, then we deduce

$$
N /(N \cap \mathfrak{P} G) \mathfrak{w} G /(N \cap \mathfrak{P} G)
$$

from (v.a) and Lemma 5.1. Combine condition (v.b) with Corollary 3.3 to show the validity of the following property:

(8) If $S_{i}$ is a normally descending sequence of principal subgroups of $[G /(N \cap \mathfrak{P G})] /\left([N /(N \cap \mathfrak{P} G)] \cap \mathfrak{S}_{3}[G /(N \cap \mathfrak{P} G)]\right)$ with

$$
S_{1} \subseteq[N /(N \cap \mathfrak{P} G)] /\left([N /(N \cap \mathfrak{P} G)] \cap \mathfrak{S}_{3}[G /(N \cap \mathfrak{P G})]\right),
$$

then $S_{k}=S_{k+1}$ for some $k$.

The properties (7) and (8) show that condition (iii) of Theorem 4.7 is satisfied by $N /(N \cap \mathfrak{B G}) \triangleleft G /(N \cap \mathfrak{P G})$. It follows that

$$
N /(N \cap \mathfrak{P} G) \subseteq \mathfrak{S}_{3}[G /(N \cap \mathfrak{P} G)] .
$$

Hence condition $\left(\mathrm{i}^{*}\right)$ is a consequence of $(\mathrm{v})$, proving the equivalence of conditions (i)-(v).

If condition (i) is satisfied by $N \triangleleft G$, then

$$
\begin{gathered}
\mathfrak{c}_{\mathrm{t} N}[(N \cap \mathbb{Q} G) /(\mathrm{t} N \cap \mathfrak{P} G)] \subseteq N \subseteq \mathfrak{Q} G \text { and } \\
N /(N \cap \mathfrak{Q} G)=1 \subseteq \mathfrak{P}[G /(N \cap \mathfrak{Q} G)] .
\end{gathered}
$$

Hence (vi) is a consequence of (i).-Assume conversely the validity of condition (vi). Let $G^{*}=G /(N \cap \mathfrak{P G})$ and $N^{*}=N /(N \cap \mathfrak{P G})$. Then we deduce from (0) that

$$
\mathrm{t} N^{*}=\mathrm{t} N /(N \cap \mathfrak{P G}) \text { and } N \cap \mathfrak{P G}=\mathrm{t} N \cap \mathfrak{P G} ;
$$

and it follows from Corollary 3.3, (a) that

$$
\left\{\begin{array}{l}
N^{*} \cap \mathfrak{H}_{3} G^{*}=(N \cap \mathfrak{Q} G) /(\mathrm{t} N \cap \mathfrak{P} G), \\
\mathrm{t} N^{*} \cap \mathfrak{S}_{3} G^{*}=(\mathrm{t} N \cap \mathfrak{Q} G) /(\mathrm{t} N \cap \mathfrak{B} G) .
\end{array}\right.
$$

It follows from (9) and (vi.a) that

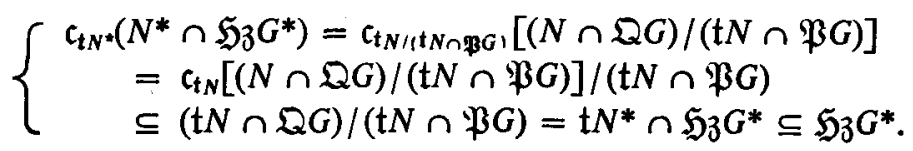


Likewise we derive irom Corollary $3.3,(b+c)$ that

$$
N^{*} /\left(N^{*} \cap \mathfrak{S}_{3} G^{*}\right) \cong N /(N \cap \mathbb{Q} G), G^{*} /\left(N^{*} \cap \mathfrak{J}_{3} G^{*}\right) \cong G /(N \cap \mathbb{Q} G)
$$

Since $N /(N \cap \mathbb{Q} G) \subseteq \mathfrak{P}[G /(N \cap \mathbb{Q} G)]$ by (vi.b), we conclude that

$$
N^{*} /\left(N^{*} \cap \mathfrak{H}_{3} G^{*}\right) \subseteq \mathfrak{P}\left[G^{*} /\left(N^{*} \cap \mathfrak{H}_{3} G^{*}\right)\right] .
$$

Because of (10) and (11) condition (v) of Theorem 4.7 is satisfied by $N^{*} \triangleleft G^{*}$ Hence $N^{*} \subseteq \mathfrak{S}_{3} G^{*}$ so that by (9)

$$
N /(\mathrm{t} N \cap \mathfrak{P G})=N^{*}=N^{*} \cap \mathfrak{S}_{3} G^{*}=(N \cap \mathbb{Q} G) /(\mathrm{t} N \cap \mathfrak{B} G) .
$$

Consequently

$$
N=N \cap \mathbb{Q} G \subseteq \mathbb{Q} G
$$

We have derived (i) from (vi), proving the equivalence of conditions (i)-( $i^{i}$

(vii) is a consequence of (vi), since (i) implies (iii.a).

ssume next the validity of (vii); and let

$$
W=\mathfrak{c}_{\mathrm{t} N}[(N \cap \mathbb{Q} G) /(\mathfrak{t} N \cap \mathfrak{P} G)]
$$

Clearly

$$
W \triangleleft G \text { and } \mathrm{t} N \cap \mathscr{P} G \subseteq W \subseteq \mathrm{t} N .
$$

It is a consequence of (vii.a) that

$$
W \mathfrak{w}_{\infty} G .
$$

Furthermore we deduce from (vii.b) that

$$
W /(W \cap \mathbb{Q} G) \cong W \mathfrak{Q} G / \mathfrak{Q} G) \subseteq N \mathfrak{Q} G / \mathfrak{Q} G \cong N /(N \cap \mathbb{Q} G) \subseteq \mathfrak{P}[G /(N \cap \mathfrak{Q} G)]
$$

Since both these isomorphisms are $G$-isomorphisms, we conclude that $W /(W \cap \Omega G)$ is a product of finite normal subgroups of $G /(W \cap \mathbb{Q} G)$. Consequently

$$
W /\left(W \cap \mathfrak{X}_{G}\right) \subseteq \mathfrak{P}[G /(W \cap \mathbb{Q} G)]
$$

If $S$ is a principal subgroup of $G /(W \cap \mathbb{Q} G)$ which is part of $W /(W \cap \mathbb{Q} G)$, then we deduce from (12.c) and the principality of $S$ the finiteness of $S$; and this implies in particular:

(12.d) If $S_{i}$ is a normally descending sequence of principal subgroups of $G /(W \cap \Omega G)$ with $S_{1} \subseteq W /(W \cap \Omega G)$, then $S_{k}=S_{k+1}$ for some $k$.

It is the content of $(12 . b+d)$ that condition $(v)$ is satisfied by $W \triangleleft G$. Hence $W \subseteq \mathfrak{Q} G$. Thus we have deduced condition (vi) from (vii), proving the equivalence of conditions (i)-(vii). 
Discussion 5.3: A. The example constructed in Discussion 4.8, $C$ satisfies conditions (iii.b), (iv.a), (v.b), (vi.b), (vii.b), though the normal subgroup $A$ is not part of $\mathfrak{Q} G$. Thus conditions (iii.a), (iv.b), (v.a), (vi.a), (vii.a) are indispensable; and it is of interest to note that condition (iv.b) cannot be weakened by requiring the finiteness of $A /(A \cap \mathbb{Q} G)$, instead of requiring that $A$ be generated by a finite class of conjugate elements.

B. There exist many groups $G$ with the properties:

$G / \mathfrak{B} G$ is finite, not 1 , and $3[G / \mathfrak{B} G]=1$;

every wreath product of an abelian group of Prüfer's type $p^{\infty}$ by a finite, simple, non-abelian group provides an example. If $G$ is such a group, then $\mathfrak{P} G=\mathfrak{Q} G$. Letting $N=G$, we have $G=\mathrm{t} N$ and $\mathfrak{c}_{\mathrm{t} N}(N \cap \mathbb{Q} G) \subseteq \mathfrak{Q} G$. Furthermore

$$
N /(N \cap \mathfrak{Q} G) \subseteq \mathfrak{P}[G /(N \cap \mathfrak{Q} G)] .
$$

This shows that in conditions (vi.a) and (vii.a) we cannot substitute $\mathfrak{c}_{t N}(N \cap \mathbb{Q} G$ ) for $\mathfrak{c}_{\mathrm{t} N}[(N \cap \mathbb{Q} G) /(\mathrm{t} N \cap \mathfrak{H G})]$.

COROLLARY 5.4. The following properties of the element $g \in G$ are equivalent:

(i) $g \in \mathbb{Q} G$.

(ii) If $s_{i}$ is a sequence of elements in $G$ with $g=s_{1}$ and $s_{i+1} \in\left\{s_{i} \circ G\right\}$ for every $i$, then the sequence $s_{i}$ is finitely valued.

(iii) If $s_{i}$ is a sequence of elements in $G$ with $g=s_{1}$ and $s_{i+1} \in\left\{s_{i} \circ G\right\}$ for every $i$, then $s_{h}=s_{k}$ for some $h<k$.

Proof. Assume first that $g \in \mathbb{Q} G$ and consider a sequence of elements $s_{i}$ in $G$ with $g=s_{1}$ and $s_{i+1} \in\left\{s_{i} \circ G\right\}$. Let $S_{i}=\left\{s_{i}^{G}\right\}$. Then the $S_{i}$ form a normally descending sequence of principal subgroups of $G$ with $S_{1}=\left\{g^{G}\right\} \subseteq \mathbb{Q} G$. Application of Theorem 5.2 shows that almost all the $S_{i}$ are finite. Hence the sequence of the elements $s_{i}$ is finitely valued: we have derived (ii) from (i).

It is clear that (iii) is a consequence [just a weak form] of (ii). If finally condition (iii) is satisfied by $g$, then $g$ is in particular a weak $Q$-G-element; and this implies $g \in \mathbb{Q} G$ by Theorem 3.2: we have verified the equivalence of conditions (i)-(iii).

COROLlary 5.5. If $N \triangleleft G$ and $\mathbb{F}$ is a finite set of normal subgroups of $G$ with $X \subseteq N$ and $N / \cdot X \subseteq \mathbb{Q}(G / X$ or every $X \in \mathcal{F}$, then

$$
N / \bigcap_{X \in \mathscr{Z}} X \subseteq \mathbb{Q}\left[G / \bigcap_{X \in \mathfrak{Z}} X\right]
$$

Proof. We assume without loss in generality that $1=\bigcap_{X \in \mathfrak{Z}} X$. Consider a normally descending sequence of principal subgroups $S_{i}$ of $G$ with $S_{1} \subseteq N$. If 
$X \in \mathfrak{F}$, then the $X S_{i} / X$ form a normally descending sequence of pr ncipal subgroups of $G / X$ with

$$
X S_{1} / X \subseteq N X / X=N / X \subseteq \mathfrak{Q}(G / X) .
$$

Application of Theo:em 5.2, (ii) shows that almost all $X S_{i} / X$ are finite. From $X S_{i} / X \cong S_{i} /\left(X \cap S_{i}\right)$ and the finiteness of $\mathfrak{F}$ we deduce now that

(1) there exists $i$ such that $S_{i} /\left(X \cap S_{t}\right)$ is finite for every $X \in \mathfrak{F}$.

Combine (1) with the finiteness of $\mathfrak{F}$ and Poincaré's Theorem to see that

$$
S . / \bigcap_{X \in \mathfrak{\Im}}\left(X \cap S_{i}\right) \text { is finite for some } i .
$$

But for every $i$ we have

$$
\bigcap_{X \in \mathscr{Z}}\left(X \cap S_{i}\right)=S_{i} \cap \bigcap_{X=\mathscr{8}} X=1
$$

so that the finiteness of $S_{i}$ is a consequence of (2). Thus we have shown that condition (ii) of Theorem 5.2 is satisfied by $N \triangleleft G$. Hence $N \subseteq \mathfrak{Q} G$, proving our result.

Remark. The Note to Lemma 1.6 shows the impossibility of omitting the requirement that the set $\mathfrak{F}$ be finite.

\section{The general properties of the group theoretical functions $\mathfrak{P}$ and $\mathfrak{Q}$}

If $\mathfrak{F}$ is one of the group theoretical functions $\mathfrak{z}, \mathfrak{G}\}, \mathfrak{B}, \mathfrak{Q}$, then

$$
\begin{gathered}
(\mathfrak{F} G)^{\sigma} \subseteq \mathfrak{F}\left(G^{\sigma}\right) \text { for every homomorphism } \sigma \text { of } G ; \\
U \cap \mathfrak{F} G \subseteq \mathfrak{F} U \text { for every subgroup } U \subseteq G .
\end{gathered}
$$

Furthermore it is a much used basic property of the hypercenter that

$$
\mathfrak{H}_{3}\left[G / \mathfrak{H}_{3} G\right]={ }_{3}\left[G / \mathfrak{S}_{3} G\right]=1 .
$$

Finally Corollary 5.5 expresses the [limited] residuality of $\mathfrak{Q}$.

6.4: Consider next an abelian group $R$ of Prüfer's type $p^{\infty}$ and a finite group $F$ with $3 F=1 \subset F$; and form the wreath product $G=R<F$. Then

$\mathfrak{P G}=\mathfrak{Q} G=$ basis subgroup of this wreath product so that

$$
G / \mathfrak{P} G=G / \mathfrak{Q} G \cong F \text { is finite, not } 1 \text {. }
$$

This shows the impossibility of proving that $\mathfrak{P}[G / \mathfrak{P G}]$ or $\mathfrak{P}[G / \mathfrak{Q} G]$ or $\mathfrak{Q}[G / \mathfrak{Q} G]$ equal 1.

6.5: Consider furthermore the example constructed in Discussion 4.8, C. Here 
${ }_{3} G=\mathfrak{S}_{3} G=\mathfrak{P} G=\mathfrak{Q} G=B, \mathfrak{P}[G / B]=A / B$ is finite of order the prime $p$.

This shows the impossibility of proving that $\mathfrak{P}\left[G / \mathfrak{S}_{3} G\right]=1$.

THEOREM 6.6. The following properties of $N \triangleleft G$ are equivalent:

$$
\begin{aligned}
& {[N /(N \cap \mathbb{Q} G)] \cap \mathbb{Q}[G /(N \cap \mathbb{Q} G)]=1 .} \\
& {[N /(N \cap \mathfrak{Q} G)] \cap \mathfrak{P}[G /(N \cap \mathbb{Q} G)]=1 \text {. }} \\
& {[N /(\mathrm{t} N \cap \mathfrak{Q} G)] \cap \mathfrak{P}[G /(\mathrm{t} N \cap \mathfrak{Q} G)]=1 .} \\
& {[\mathrm{t} N /(\mathrm{t} N \cap \mathbb{Q} G)] \cap \mathfrak{P}[G /(\mathrm{t} N \cap \mathfrak{Q} G)]=1 .} \\
& \text { (v) } 1=\left[(\mathrm{t} N \cap \mathbb{Q} G) \mathfrak{c}_{t N}[(\mathrm{t} N \cap \mathbb{Q} G) /(\mathrm{t} N \cap \mathfrak{P} G)] /(\mathrm{t} N \cap \mathbb{Q} G)\right] \cap \mathfrak{P}[G /(\mathrm{t} N \cap \mathbb{Q} G)] \text {. }
\end{aligned}
$$

Proof. It is clear that (i) implies (ii). Assume conversely the validity of (ii); and consider $x \in N$ with $x(N \cap \mathbb{Q} G) \in \mathfrak{Q}[G /(N \cap \mathfrak{Q} G)]$. If the $G$-commutator sequence $c_{i}$ contains $x$, then the $G /(N \cap \mathbb{Q} G)$-commutator sequence $c_{i}(N \cap \mathbb{Q} G)$ contains $x(N \cap \mathfrak{Q} G)$. From $\mathfrak{Q} / \mathfrak{P}=\mathfrak{S}_{\mathfrak{z}}$ and Proposition 1.1 we deduce that almost all $c_{i}(N \cap \mathfrak{Q} G)$ belong to $\mathfrak{P}[G /(N \cap \mathfrak{Q} G)]$. Because of $x \in N$ almost all $c_{i}(N \cap \mathbb{Q} G)$ belong to $N /(N \cap \mathbb{Q} G)$. Application of our condition (ii) shows that almost all $c_{i}(N \cap Q G)=1$ so that almost all $c_{i}$ belong to $N \cap Q G$. Application of Theorem 3.2 shows that the $G$-commutator sequence $c_{i}$ is finitely valued; and a second application of Theorem 3.2 shows that $x \in \mathfrak{Q} G$. Hence

$$
[N /(N \cap \mathbb{Q} G)] \cap \mathbb{Q}[G /(N \cap \mathbb{Q} G)] \subseteq \mathfrak{Q} G /(N \cap \mathbb{Q} G) ;
$$

and this clearly implies (i). Thus we have shown that

$$
\text { the conditions (i) and (ii) are equivalent. }
$$

It is clear that $\mathfrak{P X}$ is always a torsion group and that

$$
\mathrm{t}[N /(\mathrm{t} N \cap \mathfrak{Q} G)]=\mathrm{t} N /(\mathrm{t} N \cap \mathbb{Q} G) .
$$

Consequently

$$
\begin{aligned}
{[N /(\mathrm{t} N \cap \mathbb{Q} G)] } & \cap \mathfrak{P}[G /(\mathrm{t} N \cap \mathbb{Q} G)]= \\
& =\mathfrak{P}[G /(\mathrm{t} N \cap \mathbb{Q} G)] \cap[\mathrm{t} N /(\mathrm{t} N \cap \mathbb{Q} G)] .
\end{aligned}
$$

It is an immediate consequence of (2) that

$$
\text { the conditions (iv) and (iii) are equivalent. }
$$

Denote by $A$ and $B$ respectively the uniquely determined subgroups with $N \cap \mathbb{Q} G \subseteq A$ and $A /(N \cap \mathbb{Q} G)=[N /(N \cap \mathfrak{Q} G)] \cap \mathfrak{P}[G /(N \cap \mathfrak{Q} G)]$, $\mathrm{t} N \cap \mathbb{Q} G \subseteq B$ and $B /(\mathrm{t} N \cap \mathbb{Q} G)=[\mathrm{t} N /(\mathrm{t} N \cap \mathbb{Q} G)] \cap \mathfrak{P}[G /(\mathrm{t} N \cap \mathbb{Q} G)]$. 
We note that

$$
\begin{aligned}
& A \triangleleft G, N \cap \mathfrak{Q} G=\mathfrak{Q} G \cap A \subseteq A \subseteq N, A /(A \cap \mathbb{Q} G) \subseteq \mathfrak{P}[G /(A \cap \mathbb{Q} G)], \\
& B \triangleleft G, \mathrm{t} N \cap \mathbb{Q} G=\mathfrak{Q} G \cap B \subseteq B \subseteq \mathrm{t} N, B /(B \cap \mathbb{Q} G) \subseteq \mathfrak{P}[G /(B \cap \mathbb{Q} G)] .
\end{aligned}
$$

Consider $W \triangleleft G$ with $B \cap \mathbb{Q} G \subseteq W \subseteq B$ and finite $W /(B \cap \mathbb{Q} G)$. Then $W$ is a torsion group so that

$$
\mathrm{t} N \cap \mathbb{Q} G=\mathbb{Q} G \cap B=W \cap \mathbb{Q} G \cap B=W \cap \mathrm{t} N \cap \mathbb{Q} G=W \cap N \cap \mathbb{Q} G ;
$$

and this implies that

$W(N \cap \mathbb{Q} G) /(N \cap \mathbb{Q} G) \cong W /(W \cap N \cap \mathbb{Q} G)=W /(B \cap \mathbb{Q} G)$ is finite

It follows that

$$
W(N \cap \mathbb{Q} G) /(N \cap \mathbb{Q} G) \subseteq[N /(N \cap \mathbb{Q} G)] \cap \mathfrak{P}[G /(N \cap \mathbb{Q} G)]
$$

Hence $W \subseteq A$; and since $W$ is a normal torsion subgroup of $G$, it follows that $W \subseteq \mathrm{t} A$. Recalling that $B$ is by its very definition the product of such normal subgroups $W$, we have shown that

$$
B \subseteq \mathrm{t} A \text {. }
$$

We note next that

$$
\begin{aligned}
\mathrm{t} A /(\mathrm{t} A \cap \mathbb{Q} G) & =\mathrm{t} A /[\mathrm{t} A \cap(A \cap \mathbb{Q} G)] \\
& \cong \mathrm{t} A(A \cap \mathbb{Q} G) /(A \cap \mathbb{Q} G) \subseteq \mathfrak{P}[G /(A \cap \mathbb{Q} G)] .
\end{aligned}
$$

Thus $\mathrm{t} A /(\mathrm{t} A \cap \mathbb{Q} G)$ is a product of finite normal subgroups of $G /(\mathrm{t} A \cap \mathbb{Q} G$ Consider therefore $V \triangleleft G$ with

$$
\mathrm{t} A \cap \mathbb{Q} G \subseteq V \subseteq \mathrm{t} A \text { and finite } V /(\mathrm{t} A \cap \mathbb{Q} G)
$$

Then $\mathrm{t} A \cap \mathbb{Q} G=V \cap \mathrm{t} A \cap \mathbb{Q} G \subseteq V \cap \mathrm{t} N \cap \mathbb{Q} G$ so that

$$
V(\mathrm{t} N \cap \mathbb{Q} G) /(\mathrm{t} N \cap \mathbb{Q} G) \cong V /(V \cap \mathrm{t} N \cap \mathbb{Q} G) \text { is finite }
$$

as an epimorphic image of $V /(\mathrm{t} A \cap \mathbb{Q} G)$. Furthermore

$$
\mathrm{t} N \cap \mathbb{Q} G \subseteq V(\mathrm{t} N \cap \mathbb{Q} G) \subseteq \mathrm{t} A(\mathrm{t} N \cap \mathbb{Q} G) \subseteq \mathrm{t} N ;
$$

and this proves that $V \subseteq B$. From these considerations it follows that

$$
\mathrm{t} A \subseteq B .
$$

Combining (4.a) and (4.b) we see that

$$
\mathrm{t} A=B .
$$

From $A /(A \cap \mathbb{Q} G) \subseteq \mathfrak{P}[G /(A \cap \mathbb{Q} G)]$ we see that $A \triangleleft G$ satisfies condition 
(vi.b) of Theorem 5.2. Consequently $A \subseteq \mathbb{Q} G$ and $t A \subseteq \mathbb{Q} G$ are equivalent properties of $A \triangleleft G$. Combine this with (4) to see that

$$
A \subseteq \mathfrak{Q} G \text { if, and only if, } B \subseteq \mathbb{Q} G \text {. }
$$

It is an immediate consequence of the definitions of $A$ and $B$ that $A \subseteq \mathfrak{Q} G$ is equivalent with condition (ii); and that $B \subseteq \mathbb{Q} G$ is equivalent with condition (iv). Thus it follows from (5) that

$$
\text { conditions (ii) and (iv) are equivalent. }
$$

Combination of (1), (3) and (6) shows that

$$
\text { conditions (i)-(iv) are equivalent. }
$$

We recall that in the course of proving (7) we have shown that the conditions (i)-(iv) are equivalent with $B \subseteq \mathbb{Q} G$. We note next that because of $B /(B \cap \mathfrak{Q} G) \subseteq \mathfrak{P}[G /(N \cap \mathfrak{Q} G)]$ condition (vi.b) of Theorem 5.2 is satisfied by $B \triangleleft G$. It follows that the conditions

$$
\text { (i)-(iv), } B \subseteq \mathbb{Q} G \text { and } c_{t B}[(B \cap \mathbb{Q} G) /(\mathrm{t} B \cap \mathfrak{P} G)] \subseteq \mathfrak{Q} G
$$

are equivalent.

Since $B$ is by (4) a torsion group, $B=\mathrm{t} B$ and

$$
\mathrm{t} B \cap \mathbb{Q} G=B \cap \mathfrak{Q} G=\mathrm{t} N \cap \mathbb{Q} G, \mathrm{t} B \cap \mathfrak{P} G=B \cap \mathfrak{P} G=\mathrm{t} N \cap \mathfrak{P} .
$$

Hence it follows from (8) that the conditions (i)-(iv) are equivalent with

$$
\begin{aligned}
1 & =(\mathrm{t} B \cap \mathfrak{Q} G) \mathfrak{c}_{\mathrm{t} B}[(B \cap \mathfrak{Q} G) /(\mathrm{t} B \cap \mathfrak{P G})] /(\mathrm{t} B \cap \mathbb{Q} G) \\
& =\left[(\mathrm{t} N \cap \mathfrak{Q} G) \mathfrak{c}_{G}[(\mathrm{t} N \cap \mathfrak{Q} G) /(\mathrm{t} N \cap \mathfrak{P} G)] /(\mathrm{t} N \cap \mathbb{Q} G)\right] \cap[B /(\mathrm{t} N \cap \mathfrak{Q} G)] \\
& =\left[(\mathrm{t} N \cap \mathfrak{Q} G) \mathfrak{c}_{\mathrm{t}_{N}}[(\mathrm{t} N \cap \mathfrak{Q} G) /(\mathrm{t} N \cap \mathfrak{P} G)] /(\mathrm{t} N \cap \mathfrak{Q} G)\right] \cap \mathfrak{P}[G /(\mathrm{t} N \cap \mathfrak{Q} G)] \\
& =\left[(\mathrm{t} N \cap \mathfrak{Q} G) \mathfrak{c}_{\mathrm{t}_{N}}[(\mathrm{t} N \cap \mathfrak{Q} G) /(\mathrm{t} N \cap \mathfrak{P} G)] /(\mathrm{t} N \cap \mathfrak{Q} G)\right] \cap \mathfrak{Q}[G /(\mathrm{t} N \cap \mathfrak{Q} G)]
\end{aligned}
$$

and this completes the proof of the equivalence of conditions (i)-(iv) and (v).

Corollary 6.7. If $N \triangleleft G$ and $N \cap \mathfrak{B}$ is finite, then $\mathrm{t} N \cap \mathbb{Q} G$ is finite and $[N /(N \cap \mathfrak{Q} G)] \cap \mathfrak{Q}[G /(N \cap \mathfrak{Q} G)]=1$.

PRoof. Assume by way of contradiction that $N \cap \mathfrak{B G} \neq \mathrm{t} N \cap \mathbb{Q} G$. Since $N \cap \mathfrak{B G} \perp \mathrm{s}$ a torsion group, it follows that

$$
N \cap \mathfrak{P G}=\mathrm{t} N \cap \mathfrak{P G} \subset \mathrm{t} N \cap \mathbb{Q} G .
$$

From $Q / P=\mathfrak{S}_{3}$ we deduce that

$$
1 \subset[(\mathrm{t} N \cap \mathbb{Q} G) /(\mathrm{t} N \cap \mathfrak{P G})] \cap 3[G /(\mathrm{t} N \cap \mathfrak{P G})] .
$$

Since every torsion element in the center generates a finite normal subgroup, it 
follows that

(2) $[(\mathrm{t} N \cap \mathfrak{Q} G) /(\mathrm{t} N \cap \mathfrak{P G})] \cap 3[G /(\mathrm{t} N \cap \mathbb{Q} G)] \subseteq \mathfrak{P}[G /(\mathrm{t} N \cap \mathfrak{P} G)]$.

But $\mathrm{t} N \cap \mathfrak{P G}=\mathfrak{P} G \cap N$ is finite by hypothesis; and this implies

$$
\mathfrak{P}[G /(\mathrm{t} N \cap \mathfrak{P} G)]=\mathfrak{P} G /(\mathrm{t} N \cap \mathfrak{P} G / .
$$

Combining (1)-(3) we see that

$$
\begin{aligned}
1 \subset[(\mathfrak{t} N & \cap \mathbb{Q} G) /(\mathfrak{t} N \cap \mathfrak{P} G)] \cap 3[G /(\mathfrak{t} N \cap \mathfrak{P} G)] \\
& \subseteq[(\mathfrak{t} N \cap \mathbb{Q} G) /(\mathfrak{t} N \cap \mathfrak{P} G)] \cap[\mathfrak{P} G /(\mathrm{t} N \cap \mathfrak{P} G)]=1,
\end{aligned}
$$

a contradiction proving that

$$
N \cap \mathfrak{P} G=\mathrm{t} N \cap \mathbb{Q} G \text { is finite. }
$$

From (3), (4) one deduces readily that

$$
\mathfrak{P}[G /(\mathrm{t} N \cap \mathbb{Q} G)]=\mathfrak{P} G /(\mathrm{t} N \cap \mathbb{Q} G) ;
$$

and combining (4) and (5) we find that

$[\mathrm{t} N /(\mathrm{t} N \cap \mathbb{Q} G)] \cap \mathfrak{P}[G /(\mathrm{t} N \cap \mathbb{Q} G)] \subseteq[N /(N \cap \mathfrak{P} G)] \cap[\mathfrak{P} G /(N \cap \mathfrak{P} G)]=1$.

Hence condition (iv) of Theorem 6.6 is satisfied by $N$ so that

$$
[N /(N \cap \mathbb{Q} G)] \cap \mathbb{Q}[G /(N \cap \mathbb{Q} G)]=1 .
$$

Proposition 6.8. (A) The following properties of the group $G$ are equivalent:

$$
\begin{aligned}
& \mathfrak{Q}[G / \mathfrak{Q} G]=1 . \\
& \mathfrak{P}[G / \mathfrak{Q} G]=1 . \\
& \mathfrak{P}[G / \mathfrak{t Q} G]=1 . \\
& {\left[\mathrm{tQ} G \mathrm{c}_{\mathrm{t} G}(\mathrm{t} \mathfrak{Q} / \mathfrak{P} G) / \mathrm{tQ} G\right] \cap \mathfrak{P}[G / \mathfrak{t Q} G]=1 .}
\end{aligned}
$$

(B) If $\mathfrak{P G}$ is finite, then $\mathfrak{1} Q G$ is finite and $\mathbb{Q}[G / \mathfrak{Q} G]=1$.

This result is obtained by letting $N=G$ in Theorem 6.6 and Corollary 6.7.

\section{Q-groups and locally $\mathfrak{Q}$-groups}

A $Q$-Group is a group $G$ with $G=\mathfrak{Q} G$. It is, by Theorem 3.2, a group all of whose elements are $\mathbb{Q}$ - $G$-elements [weak $\mathfrak{Q}$ - $G$-elements]. It is readily seen that subgroups and epimorphic images of Q-groups are Q-groups. That extensions of $\mathbb{Q}$-groups by $\mathfrak{Q}$-groups need not be $\mathbb{Q}$-groups, has already been pointed out in section 4 . The following example shows the absence of a further closure property.

EXAmPLE 7.1. Denote by $p$ an odd prime and by $N$ a group, generated by 
elements $a_{i}, b_{j}, c_{i j}$ where $i$ and $j$ range over all the integers subject to the following relations:

$A=\left\{a_{v}, c_{i j}\right.$ for all $i$ and $\left.j\right\}$ is an elementary abelian $p$-group;

$B=\left\{b_{j}, c_{i j}\right.$ for all $i$ and $\left.j\right\}$ is an elementary abelian $p$-group;

$$
a_{i} \circ b_{j}=c_{i j} \text { for every } i \text { and } j \text {. }
$$

One checks easily that $z N=N^{\prime}=\left\{c_{i j}\right.$ for all $i$ and $\left.j\right\}$, that $A \triangleleft N$ and $B \triangleleft N$ and $N=A B$. In particular $N$ is a nilpotent $p$-group of class 2 .

There exist uniquely determined automorphisms $\sigma^{\prime}$ and $\sigma^{\prime \prime}$ of $N$, defined by the following rules:

$$
\begin{aligned}
& a^{\sigma^{\prime}}=a^{-1} \text { for } a \in A, b_{j}^{\sigma^{\prime}}=b_{j} \text { for every } j \\
& b^{\sigma^{\prime \prime}}=b^{-1} \text { for } b \in B, a_{i}^{\sigma^{\prime \prime}}=a_{i} \text { for every } i .
\end{aligned}
$$

Both these automorphisms of $N$ have order 2; they commute; and the group $\theta$ of automorphisms of $N$, generated by $\sigma^{\prime}$ and $\sigma^{\prime \prime}$, is elementary abelian of order 4 .

Let $G=N \theta$ be the product of $N$ and $\theta$, formed in the holomorph of $N$. Then $G^{\prime}=N$ and hence $G^{\prime \prime \prime}=1$ and $\mathfrak{Q} G=\mathfrak{P B}={ }_{3} N$. Let $A^{*}=\left\{A, \sigma^{\prime}\right\}$ and $B^{*}=\left\{B, \sigma^{\prime \prime}\right\}$. These are normal $Q$-subgroups of $G$ with $G=A^{*} B^{*}$.

Denote by $S$ the normal subgroup of $G$, spanned by $\sigma^{\prime} \sigma^{\prime \prime}$. One verifies successivily that $S$ contains every $a_{i}$ and every $b_{j}$ and hence every $c_{i j}$. Consequently $S=\left\{N, \sigma^{\prime} \sigma^{\prime \prime}\right\}$. Then

$$
\mathfrak{Q} S=\mathfrak{P S}=\mathfrak{z}^{S}=\mathfrak{z}^{N}=N^{\prime},
$$

showing that $\sigma^{\prime} \sigma^{\prime \prime}$ is not contained in a normal $Q$-subgroup of $G$, though $\sigma^{\prime}$ as well as $\sigma^{\prime \prime}$ is contained in a normal Q-subgroup of $G$ : the set of elements in $G$, contained in normal $Q$-subgroups of $G$, is not a subgroup of $G$.

Basic for our discussion of locally Q-groups is the following description of the noetherian $\mathbf{Q}$-groups.

Proposition 7.2. The following properties of the group $G$ are equivalent:

(i) $G$ is noetherian, $\mathfrak{I} G=\mathfrak{P} G$ is finite and $G / \mathfrak{I} G$ is nilpotent.

(ii) $\quad G$ is a finitely generated $\mathbf{Q}$-group.

(iii) $G$ is a $Q$-group and the maximum condition is satisfied by the normal subgroups of $G$.

(iv) $\quad G$ is a $\mathbb{Q}$-group whose abelian subgroups are finitely generated.

Proof. It is clear that (i) implies each of the conditions (ii)-(iv).

If $G$ is a finitely generated $Q$-group, then $G / \mathfrak{B G}$ is a finitely generated hypercentral group; and such a group is noetherian and hence nilpotent; see Baer $[1$; p. 203, Theorem]. Since $G / \mathfrak{P G}$ is noetherian and nilpotent, $G / \mathfrak{P G}$ is finitely 
presentable. Since $G$ is finitely generated, $\Re G$ is spanned by finitely many classes of conjugate elements; see Baer $[4 ;$ p. 270 , Folgerung 1$]$. Since $\mathfrak{P} G$ is the product of all finite normal subgroups of $G$, it follows that $\mathfrak{B} G$ is finite. Hence $G$ is noetherian. Since $G / \mathfrak{P G}$ is nilpotent and noetherian, $\mathfrak{I}[G / \mathfrak{B} G]$ is a finite characteristic subgroup; see Baer $[1 ;$ p. 207 , Corollary]. Since $\mathfrak{P G}$ is finite, it follows that $\mathfrak{I}[G / \mathfrak{P} G]=1$ and $\mathfrak{P} G=\mathfrak{I} G$ : we have derived (i) from (ii).

If $G$ meets requirement (iii), then the hypercentral group $G / \mathfrak{B G}$ is nilpotent and hence noetherian; see Baer [2; p. 322, Satz 1]. The product $\mathfrak{P G}$ of finite normal subgroups is a product of finitely many finite normal subgroups. Hence $\mathfrak{P G}$ is finite and $G$ is noetherian: (ii) is a consequence of (iii).

Assume the validity of (iv). Then every abelian subgroup of the locally finite group $\mathfrak{P G}$ is finitely generated and hence finite. Application of the Theorem of P. Hall-C. R. Kuatilaka shows that $\mathfrak{P} G$ is finite. Since $G / \mathfrak{P} G$ is hypercentral, $\mathfrak{c}_{G} \mathfrak{P G} / 3 \mathfrak{P G} \cong \mathfrak{P G c}_{G} \mathfrak{P G} / \mathfrak{P G}$ is hypercentral; and $G / \mathfrak{c}_{G} \mathfrak{P G}$ is finite.

It follows that ${ }_{c_{G}} \mathfrak{P G}$ is a hypercen'ral group all of whose abelian subgroups are finitely generated. Hence $c_{G} \supsetneq G$ is noetherian; see Baer [3; p. 173, Hauptsatz 4]. But then $G$ is noetherian; and we have deduced (ii) from (iv), completing the proof.

COROLlary 7.3. If $N$ is a noetherian normal subgroup of $G$ with $N \subseteq \mathbb{Q} G$, then there exists a positive integer $k$ such that every G-commutator sequence, beginning in $N$, contains at most $k$ distinct elements.

Proof. From $N \subseteq \mathbb{Q} G$ we deduce that $N$ is a noetherian $\mathfrak{Q}$-group. Application of Proposition 7.2 shows that $\mathfrak{I N}$ is a finite characteristic subgroup of $N$. Hence $\mathfrak{I} N \triangleleft G$. From $N \subseteq \mathfrak{Q} G$ it follows that $N / \mathfrak{I} N \subseteq \mathfrak{H} 3(G / \mathfrak{I} N)$; and this implies the existence of a positive integer $c$ such that

$$
N / \mathfrak{I} N=\varkappa_{c}(G / \mathfrak{I} N)
$$

where the $z_{i}$ are the terms of the ascending central series [since $N$ is noetherian]. If $c_{i}$ is a $G$-commutator sequence with $c_{1} \in N$, then it follows that $c_{1+c} \in \mathfrak{T} N$; and consequently $c_{i} \in \mathfrak{I} N$ for $c<i$. If $t$ is the order of the finite group $\mathfrak{I} N$, then it follows that the sequence of the $c_{i}$ cannot contain more than $1+c+t$ distinct elements.

REMARK. We have just completed an investigation of normal subgroups $N \triangleleft G$ with the property that $G$-commutator sequences, beginning in $N$, contain a bounded number of distinct elements.

$\mathfrak{L Q}$-Group $=$ Locally-QQ-Group $=$ group all of whose finitely generated subgroups are $Q$-groups.

Since subgroups and epimorphic images of finitely generated $\mathfrak{Q}$-groups are, by Proposition 7.2, finitely generated $\mathfrak{Q}$-groups, subgroups and epimorphic 
images of $\mathfrak{Q Q}$-groups are likewise $\mathfrak{E Q}$-groups. If $G$ is an $\mathfrak{Q Q}$-group, then every finite subset of $\mathfrak{I} G$ generates a finite subgroup [by Proposition 7.2] so that $\mathfrak{I} G$ is a locally finite group. Every finitely generated subgroup of $G / \mathfrak{I} G$ is an epimorphic image of a finitely generated subgroup of $G$; and as such it is a torsionfree finitely generated $\mathfrak{Q}$-group and hence a torsionfree nilpotent group by Proposition $\mathbf{7 . 2}$ so that $G / \mathfrak{I} G$ is torsionfree and locally nilpotent.

THEOREM 7.4. The product $\mathfrak{E Q G}$ of all normal $\mathfrak{E Q}$-subgroups of $G$ is a characteristic $\mathbb{Q Q Q}$-subgroup of $G$.

Proof. Consider first two normal $\mathfrak{Q Q}$-subgroups $A$ and $B$ of $G$. It is a consequence of Proposition 7.2 that $A$ and $B$ are locally noetherian normal subgroups of $G$; and this implies by Baer [5; p. 353, Folgerung 1$]$ that $A B$ is a locally noetherian normal subgroup of $G$. If $S$ is a finite subset of $A B$, then there exist finite subsets $\tilde{A}$ and $\tilde{B}$ of $A$ and $B$ respectively such that $S$ is contained in $U=\{\tilde{A}, \tilde{B}\}$. Naturally $U$ is finitely generated and hence noetherian. Consequently $U \cap A$ is a noetherian normal subgroup of $U$ which contains $\tilde{A}$; and $U \cap B$ is a noetherian normal subgroup of $U$ which contains $\widetilde{B}$. Hence

$$
U=(U \cap A)(U \cap B)
$$

is the product of its normal subgroups $U \cap A$ and $U \cap B$. Since $U \cap A$ is a noetherian subgroup of the $\mathscr{Q Q}$-group $A$, it tollows from Proposit.on 7.2 that

(2) $\mathfrak{I}(U \cap A)$ is a finite characteristic subgroup of $U \cap A$ with nilpotent $(U \cap A) / \mathfrak{I}(U \cap A)$;

and likewise we see that

(3) $\mathfrak{I}(U \cap B)$ is a finite characteristic subgroup of $U \cap B$ with nilpotent $(U \cap B) / \mathfrak{I}(U \cap B)$.

From $U \cap A \triangleleft U$ we deduce that $\mathfrak{I}(U \cap A) \triangleleft U$; and likewise we see that $\mathfrak{I}(U \cap B) \triangleleft U$. Consequently

(4) $T=\mathfrak{I}(U \cap A) \mathfrak{I}(U \cap B)$ is a finite normal subgroup of $U$.

Application of (1)-(4) shows that $U / T$ is the product of its nilpotent normal subgroups $T(U \cap A) / T$ and $T(U \cap B) / T$; and it is well known that this implies the nilpotency of $U / T$. Since $T$ is finite, $U$ is a noetherian $Q$-group; and this implies that its subgroup $\{S\}$ is likewise a noetherian $\mathbb{Q}$-group. Hence $A B$ is a normal $\mathscr{Q Q}$-subgroup of $G$.

Since the product of any two normal $\mathfrak{E Q}$-subgroups is a normal $\mathfrak{L Q}$-subgroups, it follows by complete induction that

(5) every product of finitely many normal $\mathfrak{Q Q}$-subgroups is a normal QQ-subgroup.

Consider a finitely generated subgroup $V$ of $\mathscr{Q Q G}$. Then it follows from the definition of $\mathscr{Q Q} G$ that $V$ is contained in a product $W$ of finitely many normal 
$\mathfrak{Q Q}$-subgroups of $G$. It is a consequence of (5) that $W$ is a normal $\mathbb{E Q}$-subgroup of $G$ so that $V$ is a $\mathfrak{Q}$-subgroup. Hence $\mathfrak{Q} Q G$ is a characteristic $\mathfrak{Q Q}$-subgroup of $G$.

REMARK 7.5. One proves by the customary arguments that every subnormal and every accessible $\mathfrak{R Q}$-subgroup of $G$ is contained in $2 \mathbb{Q} G$.

Artinian $\mathbb{Q}$-Groups. Without much trouble it is possible to prove the following characterization of this class of groups.

A. The following properties of the group $G$ are equivalent:

(i) $G$ is an artinian $Q$-group.

(ii) $G$ is a $Q$-group and the minimum condition is satisfied by the normal subgroups of $G$.

(iii) $G$ is a $\mathfrak{Q}$-group and every abelian subgroup of $G$ is artinian.

(iv) $G / \mathfrak{B G}$ is finite and nilpotent; and there exists an artinian, abelian subgroup $A$ of $\mathfrak{P G}$ with finite $[\mathfrak{P G}: A]$.

We omit the proof; its principal tools are the following results:

every locally finite artinian group contains an abelian subgroup of finite index;

every locally finite group whose abelian subgroups are artinian is artinian; see Kegel-Wehrfritz [p. 172, 5.8 Theorem].

B. Denote by $A$ an abelian group of Prüfer's type $2^{\infty}$ and let

$$
G=\left\{A, b ;(a b)^{2}=1 \text { for every } a \in A\right\} .
$$

Then $G$ is hypercentral so that $G$ is certainly a $Q$-group; and $G$ is cleariy artinian. If $a$ is an element of order $2^{n}$, then the commutator sequence $c_{i}$, defined inductively by the rules:

$$
c_{1}=a, c_{i+1}=c_{i} \circ b
$$

is readily seen to contain $n+1$ distinct elements; and this shows that the $G$ commutator sequences in art.nian Q-groups need not be bounded.

\section{Finitely valued $N-G$-commutator sequences}

An $N$-G-Commutator Sequence is for $N \triangleleft G$ a sequence of elements $c_{i} \in G$ with $c_{i+1} \in c_{i} \circ N$ for every $i$.

The terms of an $N$-G-commutator sequence belong, with the possible exception of the first term, all to $N$. Thus the terms $c_{2}, c_{3}, \cdots, c_{1}, \cdots$ of an $N-G$ commutator sequence form an $\mathrm{N}$-commutator sequence.

If $N \triangleleft G$, then $Q N$ is a characteristic subgroup of $N$ and hence a normal subgroup of $G$; and this implies that $C_{G}(N / Q N)$ is likewise a well determined normal subgroup of $G$. 
From $3\left(G / \mathfrak{S}_{3} G\right)=1$ and $\mathfrak{Q} G / \mathfrak{A} G=\mathfrak{H}_{3}(G / \mathfrak{\cap G})$ we deduce that $3(G / \mathfrak{Q} G)=1$; and this implies

$$
\mathfrak{Q} N=N \cap \mathfrak{c}_{\mathrm{G}}(N / \mathfrak{Q} N) .
$$

Definition 8.2. (A) The element $g \in G$ is a $\mathfrak{Q}-N$-G-element, if every $N-G$ commutator sequence, containing $g$, is finitely valued.

(B) The element $g \in G$ is a weak $\mathfrak{Q}-N$-G-element, if no $N$-G-commutator sequence with pairwise different elements contains $g$.

All these concepts are generalizations of the concepts, previously discussed by us. For let $G=N$ : The $G-G$-commutator sequences are just the $G$-commutator sequences; the $\mathbb{Q}-G-G$-elements are the $\mathbb{Q}-G$-elements; the weak $\mathbb{Q}-G-G$-elements are the weak $\mathfrak{Q}$ - $G$-elements; and

by $(8.1)$.

$$
\mathfrak{Q}=G \cap \mathfrak{c}_{G}(G / Q G)=\mathfrak{c}_{G}(G / \mathbb{Q} G)
$$

THEOREM 8.3. If $N \triangleleft G$, then

$\mathfrak{c}_{G}(N / \mathbb{Q} N)=$ set of $\mathbb{Q}-N$-G-elements $=$ set of weak $\mathbb{Q}-N$-G-elements.

Proof. Assume first that $g \in \mathfrak{c}_{G}(N / Q N)$; and consider an $N$-G-commutator sequence $c_{i}$ with $c_{1}=g$. Then

$$
c_{2} \in c_{1} \circ N=g \circ N \subseteq \mathfrak{c}_{G}(N / Q N) \circ N \subseteq \mathfrak{Q} N
$$

so that the elements $c_{2}, c_{3}, \cdots$ form an $N$-commutator sequence whose elements belong to $Q N$. Application of Theorem 3.2 shows that the sequence $c_{2}, c_{3}, \cdots$ is finitely valued. Thus we have shown:

(1) Every element in $c_{G}(N / \mathbb{Q} N)$ is a $Q-N$ - $G$-element.

It is clear that

(2) every $\mathfrak{Q}-N$ - $G$-element is a weak $\mathfrak{Q}-N$ - $G$-element.

Consider now a weak $\mathbb{Q}-N-G$-element $w$. If $x \in N$, then $w \circ x \in N$. Consider some $N$-commutator sequence $c_{i}$ with $c_{1}=w \circ x$. Then

$$
c_{0}=w, c_{1}=w \circ x, c_{2}, c_{3}, \cdots
$$

is an $N-G$-commutator sequence, beginning with the weak $\mathfrak{Q}-N-G$-element $w$. Assume by way of contradiction that

$$
c_{i} \neq c_{j} \text { for } 0<i<j .
$$

Since $w$ is a weak $Q-N-G$-element, it follows that

Then

$$
c_{0}=c_{k} \text { for some positive } k \text {. }
$$

$$
w=c_{0}=c_{k}, c_{k+1}, c_{k+2}, \cdots
$$


is an $N$-G-commutator sequence, beginning with the weak $\mathbb{Q}-N$-G-element $w$. Hence there exist integers $i, j$ with

$$
0<k \leqq i<j \text { and } c_{i}=c_{j} \text {. }
$$

This contradicts $(+)$; and thus we have shown that

no $N$-commutator sequence with pairwise different elements contains wo $x$.

This is equivalent to saying that $w \circ x$ is a weak $\mathfrak{Q}-N$-element; and it follows from Theorem 3.2 that $w \circ x \in \mathfrak{Q} N$. We have shown therefore that $w \circ N \subseteq \mathbb{Q} N$; and this is equivalent with $w \in \mathfrak{C}_{G}(N / \mathbb{Q} N)$. Hence

(3) every weak $\mathfrak{Q}-N$ - $G$-element belongs to $\mathfrak{C}_{G}(N / \mathfrak{Q} N)$.

Our theorem is obtained by combination of (1), (2), (3).

A Generalization. Let $S_{i}$ be for every positive integer $i$ a subset of the group $G$. Then we may consider sequences of elements $c_{i}$ in $G$ with $c_{i+1} \in c_{i} \circ S_{i}$ for $i=1,2, \cdots$. One may now consider elements with the property that every such sequence is finitely valued if it contains the given element. We have no idea how to characterize these elements, whether they form a subgroup etc. nor do we know what kind of conditions to impose upon the sequence $S_{i}$.

\section{References}

[1] Reinhold Baer, 'The hypercenter of a group', Acta Math. 89 (1953), 165-208.

[2] Reinhold Baer, 'Das Hyperzentrum einer Gruppe. III', Math. Zeitschr. 59 (1953), 292-338.

[3] Reinhold Baer, 'Auflösbare Gruppen mit Maximalbedingung', Math. Ann. 129 (1955), 139-173.

[4] Reinhold Baer, 'Noethersche Gruppen', Math. Zeitschr. 66 (1957), 269-288.

[5] Reinhold Baer, 'Lokal Noethersche Gruppen', Math. Zeitschr. 66 (1957), 341-363.

[6] P. Hall and B. Hartley, 'The stability group of a series of subgroups', Proc. London Math. Soc. 16 (1966), 1-39.

[7] P. Hall and C. R. Kulatilaka, 'A property of locally finite groups', Journal London. Math. Soc. 39 (1964), 235-239.

[3] B. Huppert, Endliche Gruppen I (Springer Verlag; Berlin-Heidelberg-New York 1967).

[9] Otto H. Kegel and Bertram A. F. Wehrfritz, Locally Finite Groups (North Holland 1973).

[10] A. G. Kurosh, The Theory of Groups. II. 2nd ed. (Translated and edited by K. A. Hirsch, Chelsea, New York, 1960).

[11] Wilhelm Specht, Gruppentheorie (Springer Verlag; Berlin-Göttingen-Heidelbərg 1956).

8053 Zürich

Eierbrechtstr. 29

Switzerland 